\title{
Characterization of Cardiac-Resident Progenitor Cells Expressing High Aldehyde Dehydrogenase Activity
}

\author{
Marc-Estienne Roehrich, ${ }^{1}$ Albert Spicher, ${ }^{1}$ Giuseppina Milano, ${ }^{2}$ and Giuseppe Vassalli, 3 \\ ${ }^{1}$ Department of Cardiology, Centre Hospitalier Universitaire Vaudois (CHUV), Avenue du Bugnon, 1011 Lausanne, Switzerland \\ ${ }^{2}$ Department of Cardiovascular Surgery, Centre Hospitalier Universitaire Vaudois (CHUV), Avenue du Bugnon, \\ 1011 Lausanne, Switzerland \\ ${ }^{3}$ Molecular Cardiology Laboratory, Fondazione Cardiocentro Ticino, Via Tesserete 48, 6900 Lugano, Switzerland \\ Correspondence should be addressed to Giuseppe Vassalli; giuseppe.vassalli@chuv.ch
}

Received 16 August 2012; Revised 29 October 2012; Accepted 2 November 2012

Academic Editor: Franca Di Meglio

Copyright (C) 2013 Marc-Estienne Roehrich et al. This is an open access article distributed under the Creative Commons Attribution License, which permits unrestricted use, distribution, and reproduction in any medium, provided the original work is properly cited.

High aldehyde dehydrogenase (ALDH) activity has been associated with stem and progenitor cells in various tissues. Human cord blood and bone marrow ALDH-bright $\left(\mathrm{ALDH}^{\mathrm{br}}\right)$ cells have displayed angiogenic activity in preclinical studies and have been shown to be safe in clinical trials in patients with ischemic cardiovascular disease. The presence of ALDH ${ }^{\text {br }}$ cells in the heart has not been evaluated so far. We have characterized $\mathrm{ALDH}^{\mathrm{br}}$ cells isolated from mouse hearts. One percent of nonmyocytic cells from neonatal and adult hearts were $\mathrm{ALDH}^{\mathrm{br}}$. $\mathrm{ALDH}^{\text {very-br }}$ cells were more frequent in neonatal hearts than adult. $\mathrm{ALDH}^{\text {br }}$ cells were more frequent in atria than ventricles. Expression of ALDH1A1 isozyme transcripts was highest in ALDH ${ }^{\text {very-br }}$ cells, intermediate in ALDH ${ }^{\text {br }}$ cells, and lowest in ALDH ${ }^{\text {dim }}$ cells. ALDH1A2 expression was highest in ALDH ${ }^{\text {very-br }}$ cells, intermediate in ALDH ${ }^{\text {dim }}$ cells, and lowest in ALDH $^{\text {br }}$ cells. ALDH1A3 and ALDH2 expression was detectable in ALDH ${ }^{\text {very-br }}$ and ALDH ${ }^{\text {br }}$ cells, unlike ALDH ${ }^{\text {dim }}$ cells, albeit at lower levels compared with ALDH1A1 and ALDH1A2. Freshly isolated ALDH ${ }^{\text {br }}$ cells were enriched for cells expressing stem cell antigen-1, CD34, CD90, CD44, and CD106. ALDH ${ }^{\text {br }}$ cells, unlike ALDH ${ }^{\mathrm{dim}}$ cells, could be grown in culture for more than 40 passages. They expressed sarcomeric $\alpha$-actinin and could be differentiated along multiple mesenchymal lineages. However, the proportion of $\mathrm{ALDH}^{\mathrm{br}}$ cells declined with cell passage. In conclusion, the cardiac-derived ALDH ${ }^{\mathrm{br}}$ population is enriched for progenitor cells that exhibit mesenchymal progenitor-like characteristics and can be expanded in culture. The regenerative potential of cardiac-derived $\mathrm{ALDH}^{\mathrm{br}}$ cells remains to be evaluated.

\section{Introduction}

Growing evidence suggests the adult heart may harbor resident stem and progenitor cells that participate in adaptive responses to myocardial injury, and possibly in cellular homeostasis under normal conditions. In most studies, the prospective isolation of cardiac stem cells has relied upon the use of specific antibodies that recognize cell-surface antigens expressed by stem cells in other tissues, particularly by hematopoietic stem cells (HSCs). Cardiac stem cells expressing stem cell antigen-1 (Sca-1) or the stem cell factor receptor, c-kit (CD117), have been described [1,2]. A distinct population of cardioblasts expressing the transcription factor islet-1 (Isl-1) and entering fully differentiated cardiomyocyte lineages has also been identified [3, 4]. However, the expression of cell-surface epitopes can vary with the metabolic state of the cell and the experimental conditions used. Therefore, no individual cell-surface antigen marker definitely identifies a single entity of cardiac stem cell. Functional properties of tissue-resident stem cells that could be used for their prospective isolation, regardless of cellsurface marker expression, have been intensely searched for. It has been proposed that, irrespective of their lineal origin, stem cells may share common mechanisms to regulate selfrenewal and differentiation, and that candidate "stemness" genes may serve as universal stem cell markers. 
Cai et al. [5] proposed that high aldehyde dehydrogenase (ALDH) activity is one of a small set of common characteristics shared by stem cells among tissues (recently reviewed by Balber [6]). Few ALDH-bright $\left(\mathrm{ALDH}^{\mathrm{br}}\right)$ pluripotential cells, measured by Aldefluor stain, gave rise to all somatic and reproductive cell lineages in tunicates [7,8]. ALDH is a cytosolic enzyme responsible for the oxidation of intracellular aldehydes. It plays important roles in oxidation of alcohol and vitamin A, and in chemoresistance to cyclophosphamide. ALDH superfamily is highly conserved across species [9]. Nineteen ALDH isoforms are known in human. $\mathrm{ALDH}^{\mathrm{br}}$, side scatter-low $\left(\mathrm{SSC}^{\mathrm{lo}}\right)$ populations from murine and human bone marrow (BM) [10-13], umbilical cord blood (UCB) [14-19], cytokine-mobilized peripheral blood [20], and circulating blood cells $[21,22]$ are enriched in stem and progenitor cells. The $\mathrm{ALDH}^{\mathrm{br}} \mathrm{SSC}^{\text {lo }}$ population includes virtually all human $\mathrm{CD} 34^{+}$and $\mathrm{CD}_{133^{+}}$cells that establish long-term, multilineage hematopoietic colonies in culture or long-term, multilineage xenografts in immunodeficient mice [16]. More recently, $\mathrm{ALDH}^{\text {br }}$ stem cells have been identified in nonhematopoietic systems, such as circulating endothelial progenitor cells (EPCs) [21,22], neural stem cells $[23,24]$, human muscle precursor cells with high myogenic activity [25, 26], colonic stem cells [27], and mammary stem cells [28]. Moreover, $\mathrm{ALDH}^{\mathrm{br}}$ cancer stem cells have been described in multiple types of cancer and shown to predict poor clinical outcomes in various contexts [27-29].

We therefore asked whether the postnatal heart harbors $\mathrm{ALDH}^{\text {br }}$ cells, as measured by Aldefluor stain. We found that the neonatal as well as the adult mouse heart harbors a relatively small population of $\mathrm{ALDH}^{\mathrm{br}}$ cells $(\approx 1 \%$ of all nonmyocytic cells present in the heart), which is enriched for cells expressing Sca-1 and other progenitor cell markers. ALDH ${ }^{\text {br }}$ cells, but not $\mathrm{ALDH}^{\mathrm{dim}}$ cells, adhered to plastic and grew in culture. The expanded population expressed sarcomeric $\alpha$ actinin, a cardiac marker, along with mesenchymal stem cell (MSC) markers. It could be induced to differentiate along multiple mesenchymal cell lineages. These results suggest that ALDH may mark a population of cardiac-resident, MSClike progenitor cells that possess superior ex vivo growth characteristics.

\section{Methods}

2.1. Mice and Cell Isolation. Neonatal (postnatal day 1), young adult (8 week-old), and aging (24 month-old) C57Bl/6 mice were purchased from Charles River Laboratories (France). Immediately after the sacrifice of the mice, the chest was opened, a canula was introduced into the left ventricular cavity, an incision was made in the right atrial wall, and the heart was perfused with heparinized PBS. The explanted heart was washed in PBS and cut into small pieces that were then placed in a Falcon tube $(50 \mathrm{~mL})$ containing $5 \mathrm{~mL}$ RPMI 1640 medium (Invitrogen, Carlsbad, CA, USA) supplemented with $12.5 \mu \mathrm{L}$ Liberase Blendzyme 4 and $25 \mu \mathrm{L}$ DNAse I (both from Roche, Basle, Switzerland), and incubated for $45 \mathrm{~min}$ at $37^{\circ} \mathrm{C}$ under gentle shaking. The cell suspension was filtered through a $70 \mu \mathrm{m}$-filter using a syringe plunger, washed in PBS, and centrifuged. The pellet was resuspended in $100 \mu \mathrm{L}$ Dead Cell Removal Kit microbeads solution (Miltenyi, Bergisch Gladbach, Germany), incubated for 15 min at RT, changed to Dead Cell Removal Buffer, and passaged through a Miltenyi LS column. The flow-through was centrifuged and the pellet was resuspended in FACS buffer. In a subset of experiments, atria and ventricles were processed separately to measure ALDH ${ }^{\text {br }}$ cells in the different cardiac chambers of origin.

2.2. Flow Cytometric Analyses. Cells were washed in PBS, resuspended in Aldefluor buffer, and then reacted with Aldefluor substrate (STEMCELL Technologies, Vancouver, BC, Canada) according to the manufacturer's instructions. After the ALDH enzyme reaction, cells were washed, resuspended in cold Aldefluor buffer, and maintained on ice during all subsequent manipulations. A forward scatter (FSC) versus side scatter (SSC) cytogram was used to gate signals from cells, and an Aldefluor fluorescence versus SSC cytogram was constructed. A unique $\mathrm{ALDH}^{\mathrm{br}}$ population was present in Aldefluor-reacted samples and absent in cells in the samples treated with the specific inhibitor of ALDH, diethylaminobenzaldehyde (DEAB). Cells incubated with Aldefluor substrate and DEAB were used to establish baseline fluorescence of these cells and to define the $\mathrm{ALDH}^{\mathrm{br}}$ and the $\mathrm{ALDH}^{\text {very-br }}$ region as less than $0.1 \%$ and $0 \%$ of total events, respectively. Cell incubation with Aldefluor substrate in the absence of inhibitor induced a shift in FL1 fluorescence defining the $\mathrm{ALDH}^{\mathrm{dim}}$, the $\mathrm{ALDH}^{\mathrm{br}}$, and the $\mathrm{ALDH}^{\text {very-br }}$ population. To assess cell-surface antigen expression, cells were incubated with antibody for $20 \mathrm{~min}$, washed, and resuspended in cold Aldefluor buffer. Flow cytometric analyses were performed on a FACSCalibur instrument (Becton Dickinson, San Jose, CA, USA) operating at $488 \mathrm{~nm}$ excitation with standard emission filters. Aldefluor fluorescence was measured in FL1 and APC in FL4. Gates used to resolve antigen-expressing cells were set using appropriate isotypematched control Abs. Data files containing at least 2,000 $\mathrm{ALDH}^{\mathrm{br}}$ cells were acquired for analysis using the CellQuest software (Becton Dickinson).

2.3. Immunostaining. Culture-expanded $\mathrm{ALDH}^{\mathrm{br}}$ cells grown in Lab-Tek chambers were fixed with $1 \%$ paraformaldehyde (PFA) for $10 \mathrm{~min}$, followed by blocking solution (1x PBS, 1\% BSA, 0,3\% Triton) for $1 \mathrm{~h}$, and incubated with rat anti-mouse c-Kit/CD117 mAb (clone 3C1; Miltenyi) coupled to APC for $1 \mathrm{~h}$ at RT, followed by goat anti-rat IgG coupled to Alexa 488 (1:400 dilution; Molecular Probes, Life Technologies, Grand Island, NY, USA). To detect type II collagen, cells were fixed in PFA, incubated in blocking solution for $1 \mathrm{~h}$, and then with mouse monoclonal anti-type II collagen 4 Abs coktail (1:100 dilution; Chondrex, Redmond, WA, USA), followed by goat anti-mouse IgG coupled to Alexa 488 (1:400). To detect sarcomeric $\alpha$-actinin, cells were fixed with $4 \%$ PFA for $1 \mathrm{~h}$, incubated in blocking solution for $1 \mathrm{~h}$, and then with mouse antisarcomeric $\alpha$-actinin mAb (1:750 dilution; 
clone EA-53, Sigma-Aldrich, St. Louis, MO, USA) at $4^{\circ} \mathrm{C}$ overnight, followed by goat anti-mouse IgG coupled to Alexa 488 ( $1: 400)$. To detect $\alpha$-smooth muscle actin ( $\alpha$-SMA), cells were stained with anti- $\alpha$-SMA rabbit polyclonal $\mathrm{Ab}$ (abcam5694; Abcam). Control sections were incubated with secondary Ab only. Nuclei were stained with Hoechst 33342 and slides were mounted with Mowiol. Pictures were taken with a Zeiss Axioplant 2 microscope (100x objective).

2.4. Antibodies. The following mAbs were used to detect the cell-surface antigen distribution of cardiac cells by flow cytometry: CD11b-APC (clone M1; Miltenyi), CD14-APC (clone Sa2-8; eBioscience; San Diego, CA, USA), CD29PE (clone RTK2758; BioLegend), CD31-APC (clone 390; eBioscience), CD34-APC (clone RAM-34; eBioscience), CD38-APC (clone 90; eBioscience), CD40-APC (clone 1C10; eBioscience), CD44-APC (clone IM7; eBioscience), CD45-APC (clone 30-F11; eBioscience), CD90.2-APC (clone HIS51; eBioscience), CD105-PE (clone MJ7/18; eBioscience), CD106-PE (clone 429; eBioscience), cKit/CD117-APC (clone 3C1; Miltenyi), CD133-APC (clone 13A4; eBioscience), CD140b-APC (eBioscience), CD146-FITC (Miltenyi), Flk-1-APC (clone Avas12a1; eBioscience), Lineage Cell Detection Cocktail-Biotin mouse (Miltenyi; cat. no. 130-092-613), MHC class II-APC (clone M5; eBioscience), NG2 chondroitin sulfate proteoglycan (Chemicon/Millipore; cat. no. AB5320), Sca-1-APC (clone D7; eBioscience), rat IgG2a isotype control-APC (clone eBR2a; eBioscience), rat IgG2a isotype control-FITC (clone eBR2a; eBioscience), rat IgG2a isotype control-PE (clone eBR2a; eBioscience), rat mouse IgG1, $\kappa$ isotype control (clone P3; eBioscience), and Arminian hamster IgG isotype control (clone eBio299Arm; eBioscience).

2.5. Real Time RT-PCR. Freshly isolated ALDH ${ }^{\mathrm{dim}}, \mathrm{ALDH}^{\mathrm{br}}$, and ALDH ${ }^{\text {very-br }}$ cells from PBS-perfused hearts from 8week-old mice $(n=4)$ were sorted using a Beckman Coulter MoFlo Astrios FACS system. Total mRNA from each cell subset was extracted using the RNeasy Micro kit (Qiagen). The different c-DNAs were generated using the Quantitect reverse transcription kit (Qiagen) from total mRNA obtained with a genomic DNA digestion step according to the manufacturer's instructions. cDNA (1:10 dilution) was used for quantification using the RT ${ }^{2}$ SYBR Green qPCR Kit (Qiagen) and the Rotor-Gene 2000 system (Qiagen) according to the manufacturer's instructions. Real-time PCR reactions (in triplicates) were set up in $10 \mu \mathrm{L}$ reaction volume with $5 \mu \mathrm{L}$ of $\mathrm{RT}^{2}$ SYBR Green mix, $0.4 \mu \mathrm{L}$ of $10 \mu \mathrm{M} \mathrm{RT}{ }^{2}$ qPCR Primer Assay, $3 \mu \mathrm{L} c \mathrm{DNA}$, and $1.6 \mu \mathrm{L}$ water. The polymerase was heat-activated for $10 \mathrm{~min}$ at $95^{\circ} \mathrm{C}$, and the reactions were then cycled 50 times $\left(95^{\circ} \mathrm{C}, 15 \mathrm{sec} ; 55^{\circ} \mathrm{C}, 40 \mathrm{sec} ; 72^{\circ} \mathrm{C}\right.$, $30 \mathrm{sec}$ ), followed by a melting step. Primers were obtained from (Qiagen). Relative expression was calculated with the comparative $\triangle \mathrm{Ct}$ method using GUSB as a reference gene.

2.6. Ex Vivo Cultures of $A L D H^{b r}$ Cells. In two preliminary experiments, cells from enzymatically dissociated atria and ventricles were reacted separately with Aldefluor, and $\mathrm{ALDH}^{\text {br }}$ cells were sorted by FACS and placed in Corning Costar 6-well plates (Sigma) with no extracellular matrix protein coating. Ventricular ALDH ${ }^{\text {br }}$ cells grew poorly in culture, possibly as a result of a very long sorting procedure due to the scarcity of $\mathrm{ALDH}^{\text {br }}$ cells in the ventricular population. Therefore, atrial cells from 8 -week-old mice were used in subsequent experiments ( $n=3$ per experiment), allowing for a marked abbreviation of the sorting procedure. ALDH ${ }^{\mathrm{br}}$ cells were cultured in MesenCult medium (MesenCult MSC Basal Medium supplemented with serum-containing MesenCult MSC Stimulatory Supplements-Mouse; STEMCELL Technologies). ALDH ${ }^{\text {dim }}$ cells were studied for comparison. To assess the impact of the culture medium on cell phenotype, $\mathrm{ALDH}^{\text {br }}$ sorted cells were also cultured in RPMI/FCS medium (RPMI 1640; Gibco, supplemented with 10\% fetal calf serum). The AlamarBlue assay (Promega, Madison, WI, USA) was used to assess cell viability and growth. In a separate experiment, cells were cultured in the presence of imatinib (methanesulfonate salt, $0.1-10 \mu \mathrm{M}$; LC Laboratories, Woburn, MA, USA), a inhibitor of receptor tyrosine kinases encoded by c-kit and platelet-derived growth factor receptor $\mathrm{B}$ (PDGFRB). Cells were incubated with imatinib for $42 \mathrm{~h}$ (in triplicates) before AlamarBlue was added. Absorbance was measured at $570 \mathrm{~nm}$ and $595 \mathrm{~nm}$ every hour during 6 hours, and AlamarBlue reduction was calculated.

\subsection{Differentiation Potential of Expanded $A L D H^{b r}$ Cells.} Cells sorted on the basis of high ALDH activity were cultured for 8 months in MesenCult, which was then replaced by $\mathrm{NH}$ ChondroDiff Medium (for 21 days), NH OsteoDiff Medium (7 days), or AdipoDiff Medium (14 days; all from Miltenyi). Chondrogenic differentiation was assessed by immunostaining for type II collagen. To assess osteogenic and adipogenic differentiation, cells were incubated with $1 \%$ PFA for $10 \mathrm{~min}$, followed by $2 \%$ Alizarin Red solution for $5 \mathrm{~min}$ and Oil Red$\mathrm{O}$ solution for $15 \mathrm{~min}$, respectively, and by $3 \mathrm{PBS}$-washes.

\section{Results}

3.1. $A L D H^{b r}$ Cells in Neonatal and Young Adult Hearts. Freshly isolated nonmyocytic cells from enzymatically dissociated, neonatal or 8-week-old hearts were analyzed by flow cytometry using the Aldefluor reagent. ALDH ${ }^{\text {br }}$ cells amounted to $1.00 \pm 0.59 \%$ of all nonmyocytic cells in the neonatal heart $(n=3)$ and to $0.99 \pm 0.55 \%$ of these cells in the young adult heart ( $n=7, \mathrm{NS}$; Figure 1$)$. Within the ALDH ${ }^{\text {br }}$ population, the percentage of $\mathrm{ALDH}^{\text {very-br }}$ cells in neonatal hearts was higher than in young adults $(52.71 \pm 6.87 \%$ versus $19.39 \pm 2.42 \% ; P<0.05)$.

3.2. $A L D H^{b r}$ Cells in Atria and Ventricles. In young adult mice, $\mathrm{ALDH}^{\text {br }}$ cells in the atrial population were more frequent than in the ventricular $(10.29 \pm 8.44 \%$ versus $1.05 \pm$ $0.78 \% ; n=4 ; P<0.05$; Figure 2). Atrial $\mathrm{ALDH}^{\mathrm{br}}$ cells predominantly exhibited SSC ${ }^{\text {lo }}$ properties but a subset 

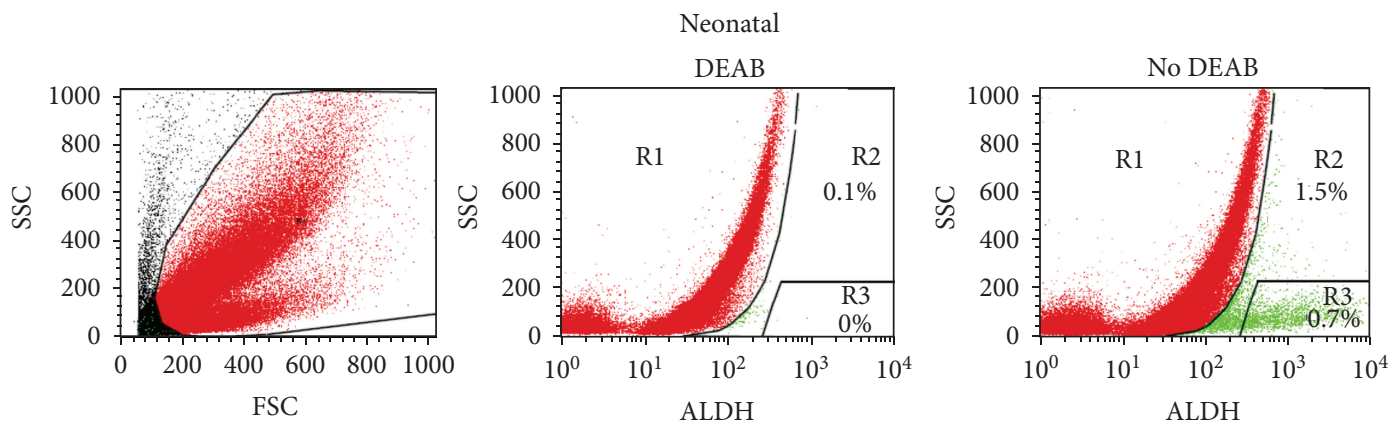

(a)
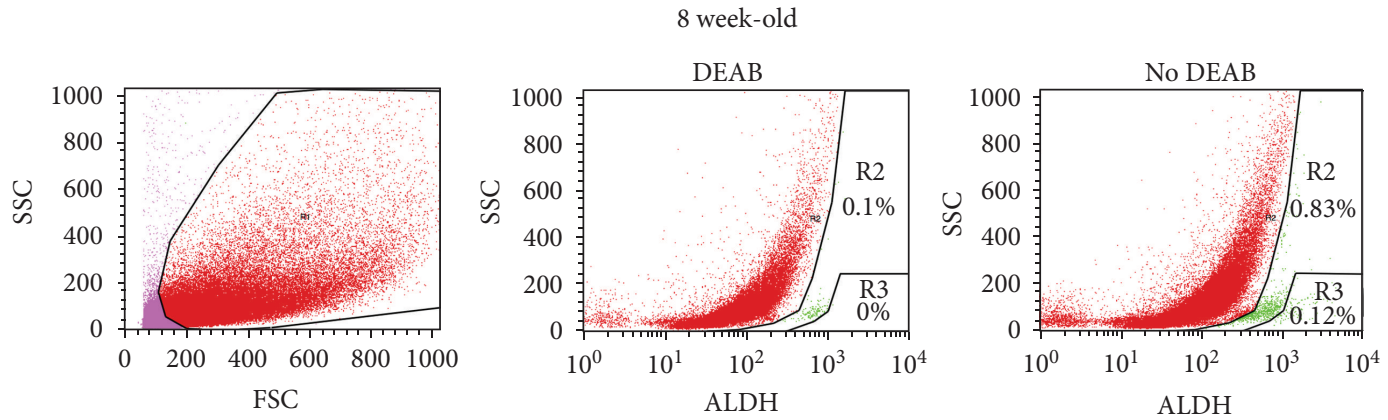

(b)

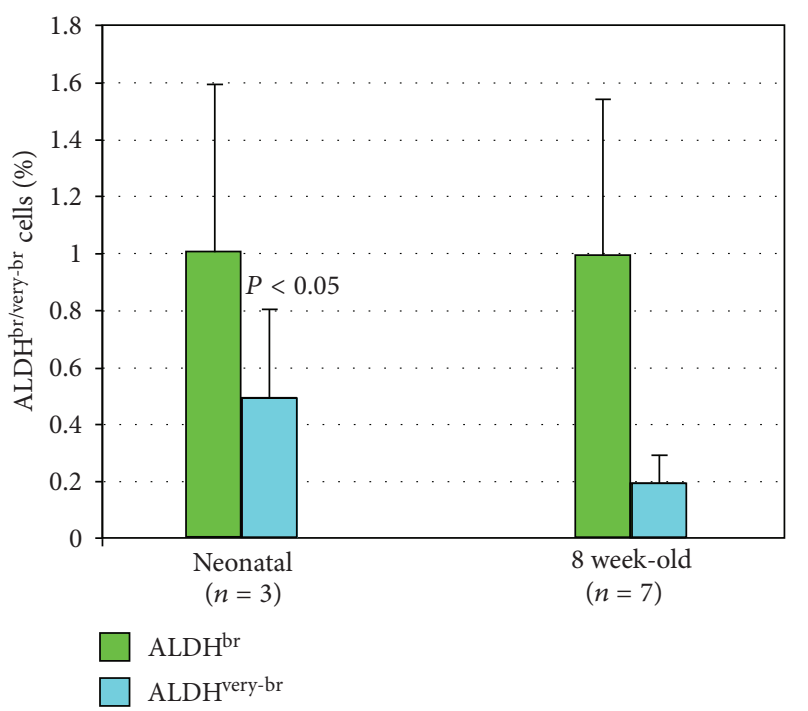

(c)

FIGURE 1: Identification of $\mathrm{ALDH}^{\text {br }}$ cells in populations isolated from whole hearts from neonatal and young adult mice. Flow cytometric analysis of ALDH activity (a). Neonatal mice. Left panel: cells were selected according to forward scatter (FSC) and side scatter (SSC) properties using the gated region. Middle panel: cells incubated with Aldefluor substrate and the specific inhibitor of ALDH, DEAB were used to establish baseline fluorescence of these cells and to define the $\mathrm{ALDH}^{\mathrm{br}}(\mathrm{R} 2)$ and the ALDH ${ }^{\text {very-br }}$ (R3) region as less than 0.1\% and 0\% of total events, respectively. Right panel: cell incubation with Aldefluor substrate in the absence of inhibitor induced a shift in FL1 fluorescence defining the $\mathrm{ALDH}^{\mathrm{dim}}$ (R1), the $\mathrm{ALDH}^{\mathrm{br}}$ (R2), and the $\mathrm{ALDH}^{\text {very-br }}$ (R3) populations (b). Eight-week-old mice (c). Bar histogram showing $\mathrm{ALDH}^{\mathrm{br}}$ and $\mathrm{ALDH}^{\text {very-br }}$ cell percentages (mean, $\mathrm{SD}$ ) in neonatal and young adult mice. $P$ value refers to the difference between neonatal and 8 week-old mice. 
showed intermediate-to-high SSC properties. Ventricular $\mathrm{ALDH}^{\text {br }}$ cells exhibited more homogeneous SSC ${ }^{\text {lo }}$ properties.

3.3. Immunophenotype of Freshly Isolated ALDH $H^{b r}$ Cells. We used a panel of mAbs to determine the cell-surface marker profile of $\mathrm{ALDH}^{\mathrm{br}}, \mathrm{ALDH}^{\text {very-br}}$, and $\mathrm{ALDH}^{\mathrm{dim}}$ cells in the freshly isolated, nonmyocytic cardiac-derived population in 8 weeks old as well as in 24 months old mice (Figure 3). Aging hearts contained $1.36 \% \mathrm{ALDH}^{\text {br }}$ cells, of which $36.8 \%$ were $\mathrm{ALDH}^{\text {very-br }}$ (pooled sample from 5 hearts). A majority of $\mathrm{ALDH}^{\text {br }}$ and $\mathrm{ALDH}^{\text {very-br }}$ cells from young adult hearts stained positive for Sca-1 $(\approx 60 \%$ and $\approx 70 \%$, resp.). Substantial subsets $(\approx 25-50 \%)$ of $\mathrm{ALDH}^{\text {br }}$ cells stained positive for CD34, CD106, CD44, CD90, and CD105 (Figure 4). The $\mathrm{ALDH}^{\mathrm{br}}$ population was significantly enriched for cells expressing Sca-1, CD90, CD34, CD106, and CD44 (foldchanges: $4.2,4.6,6.2,24.1$, and 34.3 , resp.,) whereas it was significantly depleted for cells expressing CD45 (leukocyte common antigen), CD31 (an endothelial marker), and CD38 (fold-changes: $0.5,0.36$, and 0.22 fold). The immunophenotype of ALDH ${ }^{\text {br }}$ cells derived from aging hearts was similar to that of $\mathrm{ALDH}^{\text {br }}$ cells from young adult hearts.

3.4. $m R N A$ Expression of Selected ALDH Isoforms. mRNA expression of ALDH1A1, ALDH1A2, ALDH1A3, and ALDH2 isoforms was measured by real-time RT-PCR in purified $\mathrm{ALDH}^{\mathrm{dim}}, \mathrm{ALDH}^{\mathrm{br}}$, and $\mathrm{ALDH}{ }^{\text {very-br }}$ cells (Figure 5). Gene transcripts of all of the four ALDH isoforms could be detected in $\mathrm{ALDH}^{\text {br }}$ and $\mathrm{ALDH}^{\text {very-br }}$ cells, whereas only ALDH1A1 and ALDH1A2 were detectable in ALDH ${ }^{\text {dim }}$ cells. In $\mathrm{ALDH}^{\text {br }}$ and ALDH ${ }^{\text {very-br }}$ cells, ALDH1A1 was expressed at higher levels than ALDH1A3 $\left(2^{-\Delta \mathrm{Ct}}=4.0\right.$ and 4.7, resp.,) and $\operatorname{ALDH} 2\left(2^{-\Delta \mathrm{Ct}}=4.8\right.$ and 8.8, resp.,) while ALDH1A2 was expressed at higher levels than ALDH1A3 $\left(2^{-\Delta C t}=1.7\right.$ and 12.3, resp., $)$ and ALDH2 $\left(2^{-\Delta C t}=2.1\right.$ and 23.2, resp.). In addition, ALDH1A1 was expressed at higher levels in $\mathrm{ALDH}^{\mathrm{br}}$ and $\mathrm{ALDH}^{\text {very-br }}$ cells compared to $\mathrm{ALDH}^{\mathrm{dim}}$ cells $\left(2^{-\Delta \Delta \mathrm{Ct}}=2.0\right.$ and 3.5 , resp.). ALDH1A2 expression was higher in $\mathrm{ALDH}^{\mathrm{very}-\mathrm{br}}$ cells compared to $\mathrm{ALDH}^{\mathrm{dim}}$ cells $\left(2^{-\Delta \Delta \mathrm{Ct}}=4.9\right)$ and was lowest in $\mathrm{ALDH}^{\mathrm{br}}$ cells $\left(2^{-\Delta \Delta \mathrm{Ct}}=0.5\right.$ versus $\left.\mathrm{ALDH}{ }^{\mathrm{dim}}\right)$. mRNA expression of three genes implicated in angiogenesis was also measured. $2^{-\Delta \mathrm{Ct}}$ values for $\mathrm{ALDH}^{\mathrm{dim}}, \mathrm{ALDH}^{\mathrm{br}}$, and $\mathrm{ALDH}^{\text {very-br }}$ cells were as follows: endoglin (CD105): 11.70, 10.41, and 14.86; ephrin B4: 2.98, 0.90, and 2.60; angiopoietin 1: undetectable, 0.01, and 0.008 , respectively.

3.5. Ex Vivo Culture-Expansion of $A L D H^{b r}$ Cells. ALDH ${ }^{\text {br }}$ cells were purified from the atrial population, which was enriched for these cells compared to ventricles, in order to abbreviate the FACS procedure, thereby limiting cell damage. Purified atrial ALDH ${ }^{\text {br }}$ cells grew in culture, whereas $\mathrm{ALDH}^{\mathrm{dim}}$ cells did not, even when plated at a 10-fold higher density compared to their $\mathrm{ALDH}^{\text {br }}$ counterparts (Figure $6(\mathrm{a}))$. However, $>95 \% \mathrm{ALDH}^{\mathrm{dim}}$ cells appeared to be viable, as assessed by DAPI, immediately after the FACS procedure. Atrially derived bulk populations of nonmyocytic cells grew poorly. Purified ALDH ${ }^{\text {br }}$ cells gave rise to small numbers of cell colonies, each one apparently originating from a single cell, which formed a monolayer of plastic-adherent cells, which could be expanded for more than 40 passages (later passages were not tested). In one cell culture, the percentage of $\mathrm{ALDH}^{\mathrm{br}}$ cells at passage 7 was $11 \%$; however, this parameter was not systematically measured at various time points. Growth rates in MesenCult medium were higher than in RPMI/FCS medium (Figure 6(b)). This was confirmed using the AlamarBlue assay (Figure 6(c)). Imatinib inhibited cell growth in a dose-dependent manner (Figure 6(d)).

\subsection{Immunophenotype of Culture-Expanded ALDH $H^{b r}$ Cells.}

The marker profile of expanded $\mathrm{ALDH}^{\mathrm{br}}$ cells (P11-13) was analyzed by flow cytometry (Figure 7). Cells expanded in MesenCult medium stained positive for Sca-1, CD29, CD44, CD105, CD106, and, in part, CD146 and CD14. They stained negative for CD45, CD11b, CD31, and CD133. To assess whether the culture medium affected marker expression, cells were also grown in RPMI/FCS. The immunophenotype of these cells was similar to those grown in MesenCult, although larger cell subsets stained positive for c-kit, CD140b (PDGFRB) and NG2 chondroitin sulfate proteoglycan (Figures 7(b) and 7(c)). NG2 essentially colocalized with CD140b and CD146 (Figure 7(d)). c-kit expression was demonstrated by immunocytochemistry (Figure $7(\mathrm{e})$ ).

3.7. Expanded Cells Express Sarcomeric $\alpha$-Actinin and Differentiate along Mesenchymal Lineages. Culture-expanded $\mathrm{ALDH}^{\mathrm{br}}$ cells could be induced to differentiate along adipogenic, osteogenic, and chondrogenic lineages in appropriate culture media, as evidence by staining with Oil red-O, Alizarin red, Alcian blue (not shown), and type II collagen immunostaining, respectively (Figures 8(a), 8(c) and 8(g)). Cells cultured in MesenCult stained positive for sarcomeric $\alpha$-actinin (Figure $8(\mathrm{e})$ ) but negative for $\alpha$-SMA and von Willebrand factor (not shown).

\section{Discussion}

Growing evidence suggests high ALDH activity may be a common feature shared by stem and progenitor cells across normal tissues, as well as in cancer. Human UCB and BM cells possessing high ALDH activity have shown angiogenic activity in preclinical studies [30-32] and have been used safely in phase I clinical trials in patients with ischemic cardiovascular disease [33-35].

We characterized, for the first time, $\mathrm{ALDH}^{\text {br }}$ cells isolated from the heart. Approximately one percent of all nonmyocytic cells present in the young adult mouse heart were $\mathrm{ALDH}^{\mathrm{br}}$, as measured by Aldefluor stain (Figure 1). The neonatal heart contained a similar number of $\mathrm{ALDH}^{\mathrm{br}}$ cells, although with a higher proportion of cells exhibiting very 

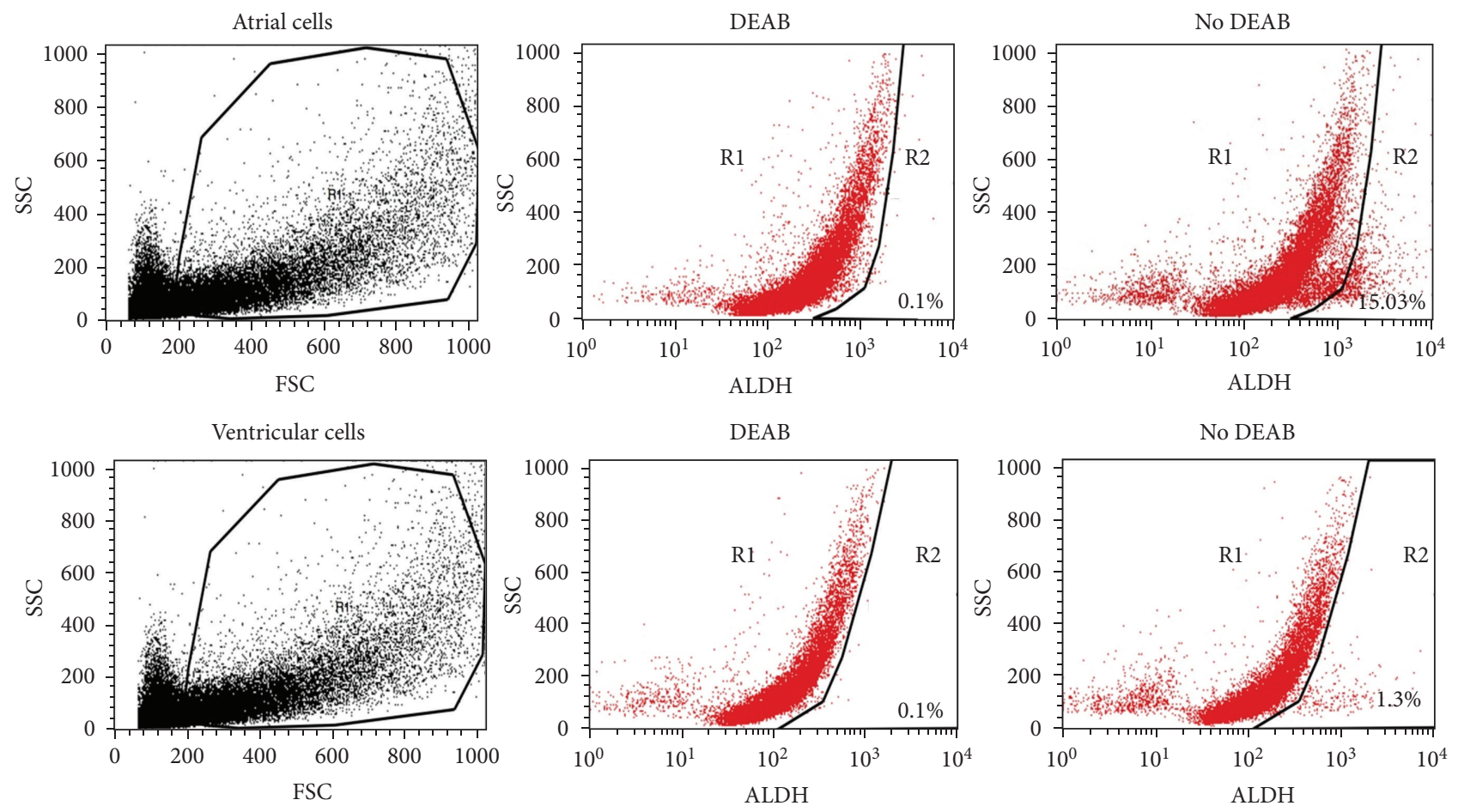

(a)

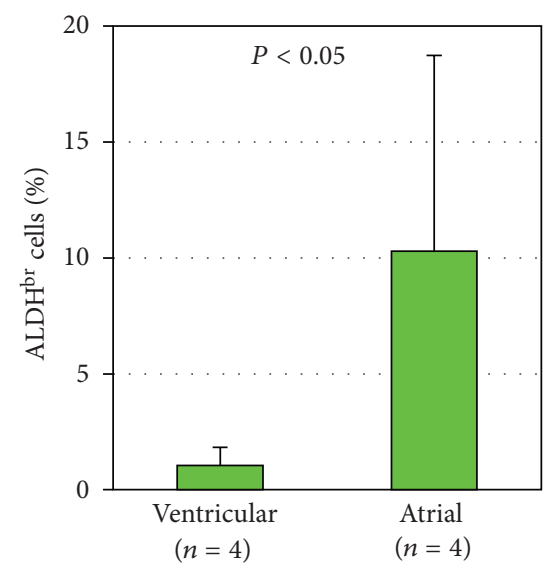

(b)

FIGURE 2: Identification of ALDH ${ }^{\text {br }}$ cells in the atrial and ventricular population from 8 week-old mice. Flow cytometric analysis of ALDH activity (a). Left panels: atrial and ventricular cells were selected according to forward scatter (FSC) and side scatter (SSC) properties using the gated region. Middle panels: cells incubated with Aldefluor substrate and the specific inhibitor of ALDH, DEAB, were used to establish baseline fluorescence of these cells and to define the $\mathrm{ALDH}^{\mathrm{br}}$ region (R2) as less than $0.1 \%$ of total events. Right panels: cell incubation with Aldefluor in the absence of inhibitor induced a shift in FL1 fluorescence defining the ALDH ${ }^{\mathrm{dim}}$ (R1) and the ALDH ${ }^{\text {br }}$ (R2) population. In the example shown, not all of the atrial cells exhibiting a shift in FL1 fluorescence in the absence of inhibitor were found in the gated region $\mathrm{R} 2$, suggesting that atrial $\mathrm{ALDH}^{\text {br }}$ cells may have been underestimated (b). Bar histogram showing percent ALDH ${ }^{\text {br }}$ cells (mean, SD) in the ventricular and atrial population.

high ALDH activity (ALDH ${ }^{\text {very-br }}$ ) compared to the young adult heart. The frequency of $\mathrm{ALDH}^{\text {br }}$ cells in the population isolated from the atria was approximately 10 -fold higher than in cells derived from ventricles (Figure 2). This observation has analogies with previous reports on increased numbers of putative stem/progenitor cells, such as DNA-label retaining cells in rodents $[36,37]$ and c-kit $^{+}$cells in human [38], in atria relative to ventricles. In the present study, $\mathrm{ALDH}^{\mathrm{br}}$ cells isolated from atria and ventricles exhibited slightly different side scatter (SSC) properties. Most atrial ALDH ${ }^{\text {br }}$ 


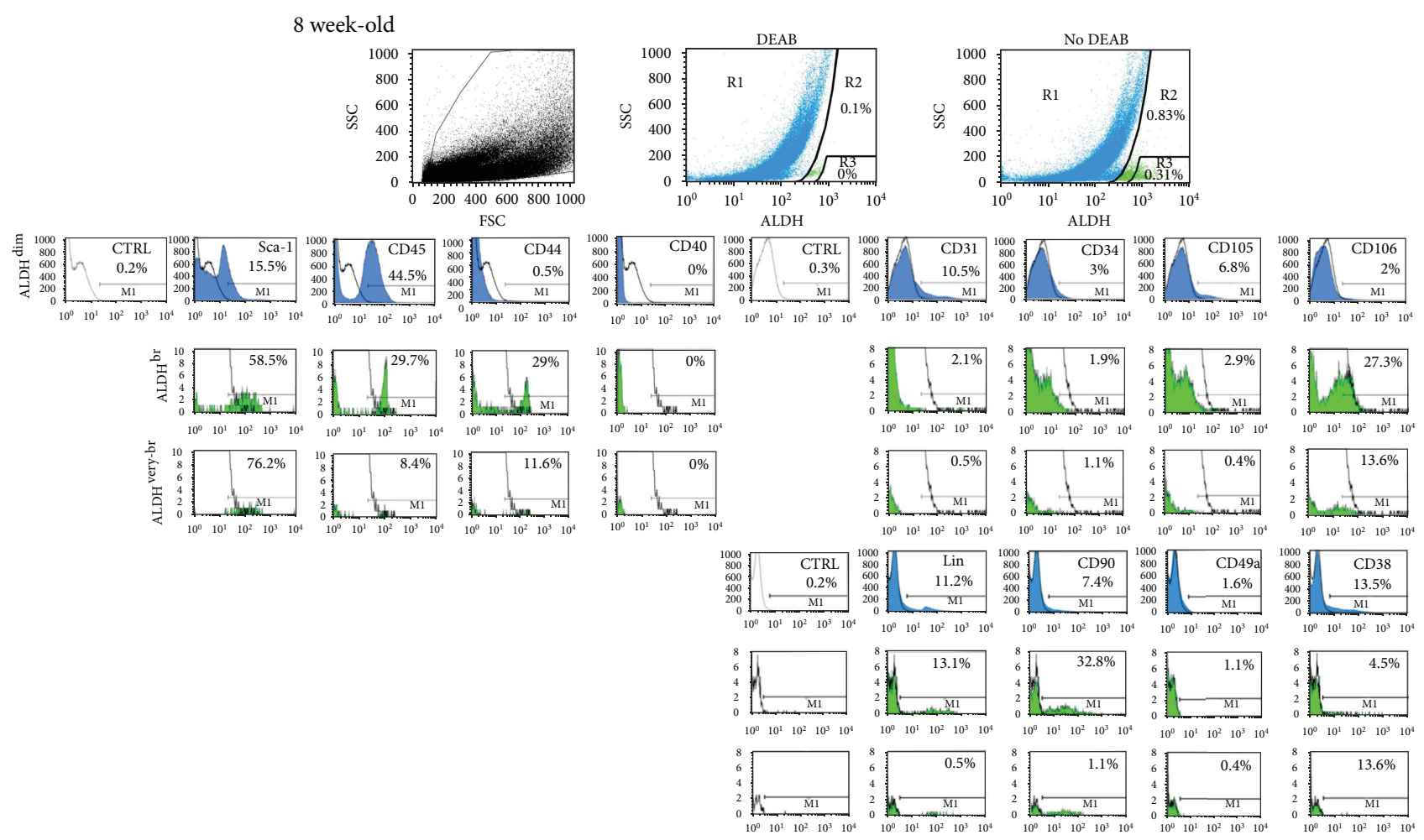

(a)

24 month-old
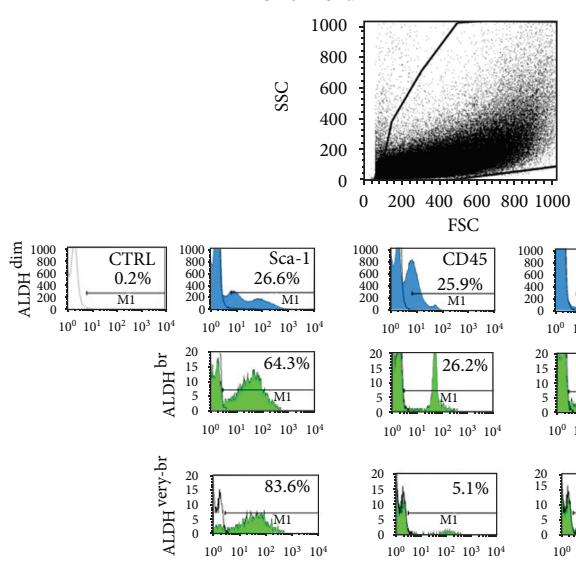
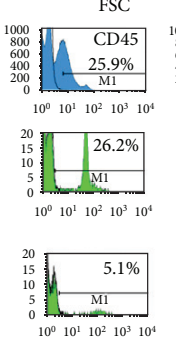
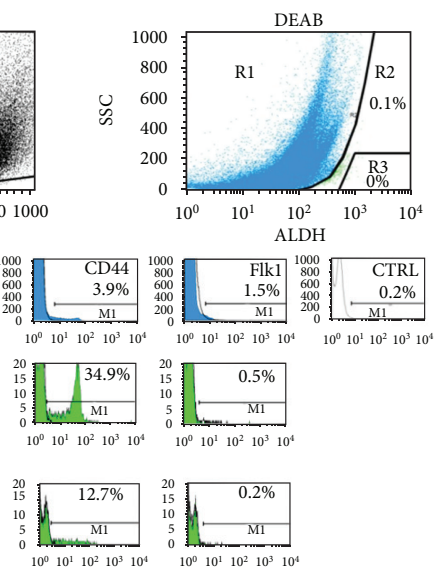
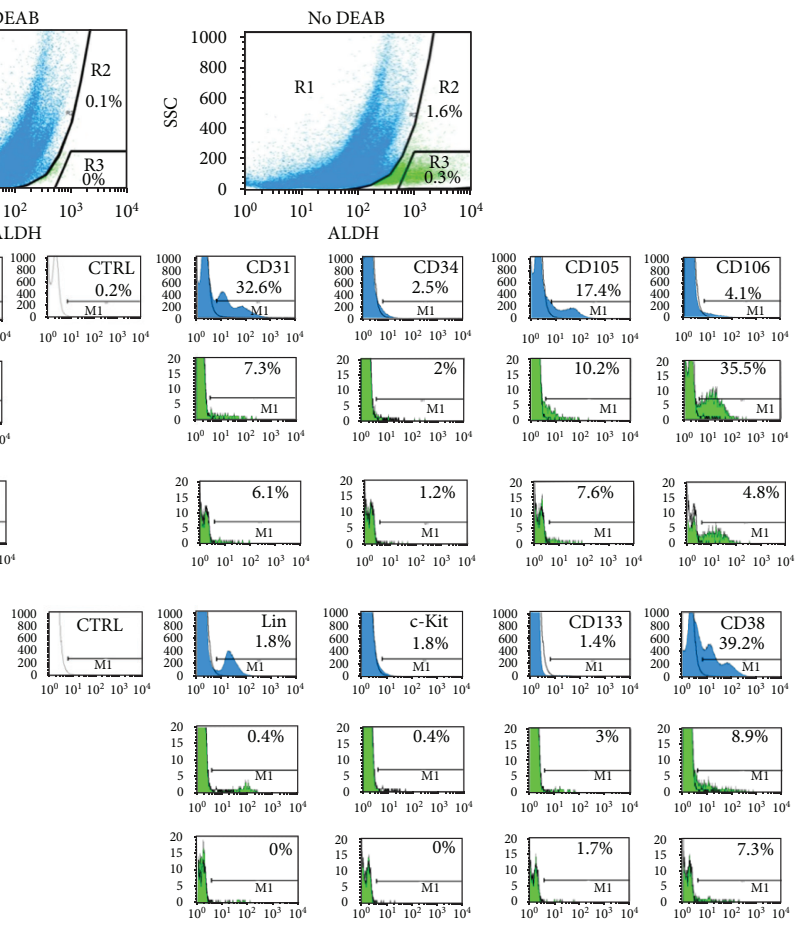

(b)

FIGURE 3: Freshly isolated ALDH ${ }^{\text {br }}$ cells from young adult hearts (upper panel; (a)) and from aging hearts (lower panel; (b)) were analyzed by flow cytometry for ALDH expression in combination with the expression of cell-surface markers. Cells were incubated with Aldefluor substrate and an inhibitor of ALDH (DEAB), or with Aldefluor alone, to establish R1, R2, and R3 gates for ALDH ${ }^{\mathrm{dim}}$ (blue), ALDH ${ }^{\text {br }}$, and $\mathrm{ALDH}^{\text {very-br }}$ cells (both green), respectively. These populations were subsequently analyzed for expression of markers. Cytograms for each surface marker in each cell population are shown (the surface markers indicated for the cytograms of ALDH ${ }^{\text {dim }}$ cells also refer to the cytograms of the respective $\mathrm{ALDH}^{\mathrm{br}}$ and $\mathrm{ALDH}{ }^{\mathrm{very}-\mathrm{br}}$ populations; corresponding cytograms are aligned vertically. Percentages of positive cells are indicated (CTRL; isotype-matched irrelevant IgG). 

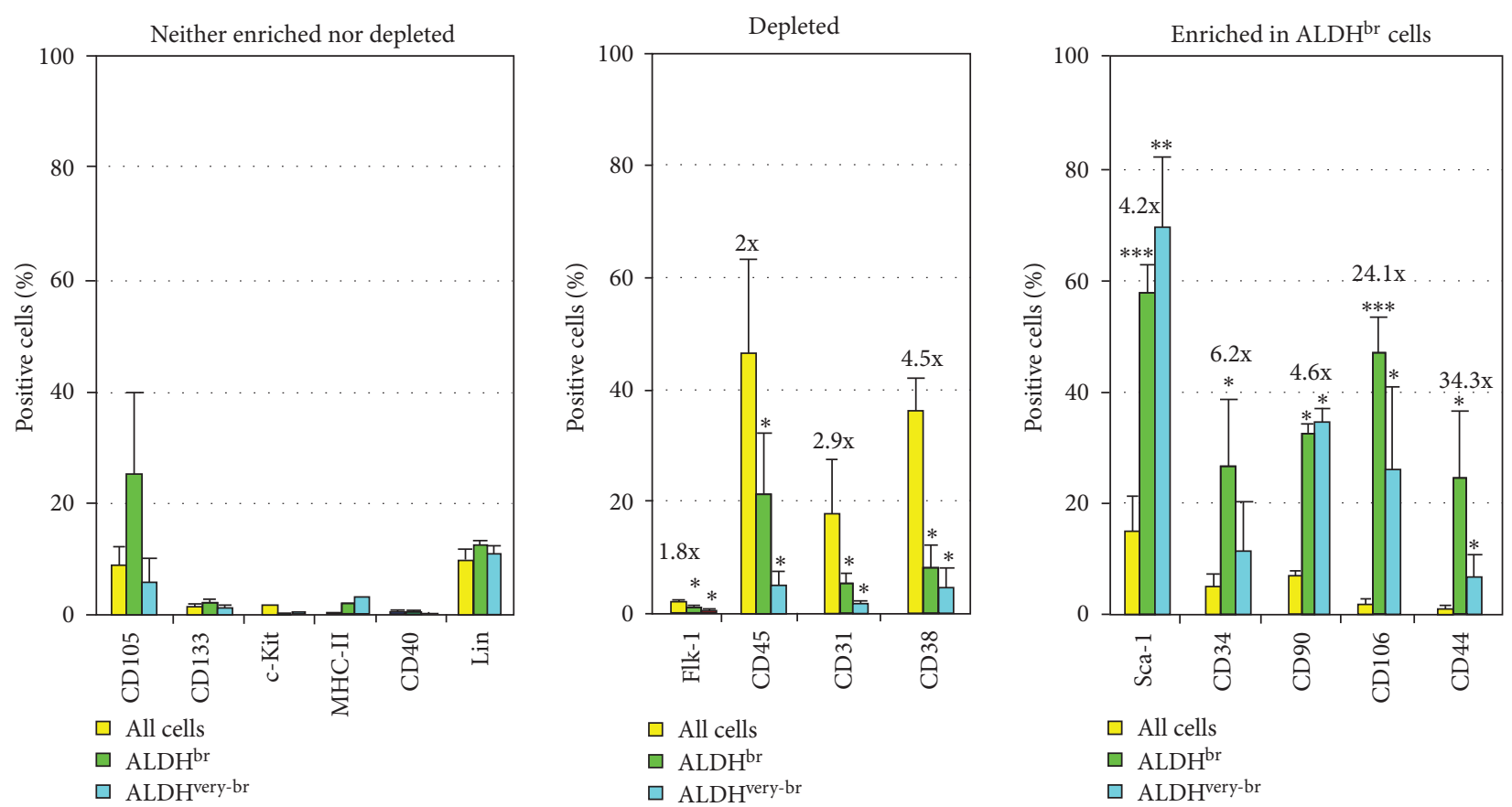

FIGURE 4: Flow cytometric analysis of the expression of cell-surface antigen markers in freshly isolated nonmyocytic cardiac cells from 8-week-old mice. Data are percentages (mean, SD) of positive cells for the indicated markers, subdivided into three groups: those significantly enriched, those significantly depleted, and those neither enriched nor depleted in the ALDH ${ }^{\text {br }}$ population. Yellow bars indicate bulk populations of cardiac-derived cells, green bars indicate $\mathrm{ALDH}^{\mathrm{br}}$ cells, and blue bars indicate ALDH ${ }^{\text {very-br }}$ cells. Numbers are enrichment/depletion factors for the indicated surface markers in the $\mathrm{ALDH}^{\text {br }}$ population $\left({ }^{* * *} P<0.001,{ }^{*} P<0.05\right.$ versus ALDH ${ }^{\mathrm{dim}}$ cells).

cells showed SSC ${ }^{\text {lo }}$ properties but a small subset possessed intermediate SSC properties. In contrast, ventricular ALDH ${ }^{\text {br }}$ cells uniformly exhibited SSC ${ }^{\text {lo }}$ properties. The original characterizations of $\mathrm{ALDH}^{\mathrm{br}}$ cells from human UCB revealed that the $\mathrm{ALDH}^{\mathrm{br}} \mathrm{SSC}^{\mathrm{lo}}$ population was enriched 50- to 100 -fold for primitive hematopoietic progenitors $[11,15]$. In several studies, SSC ${ }^{\text {lo }}$ properties therefore were associated with the traditional definition of $\mathrm{ALDH}^{\mathrm{br}}$ hematopoietic cells. However, other studies focused on $\mathrm{ALDH}^{\mathrm{br}} \mathrm{Lin}^{-}$(lineagedepleted) cells $[13,14,16]$. We defined ALDH $^{\text {br }}$ cells based solely on ALDH activity, regardless of SSC and lineage properties. This methodological approach affected ALDH ${ }^{\text {br }}$ cell counts only marginally, given the predominant $\mathrm{ALDH}^{\mathrm{br}} \mathrm{SSC}^{\mathrm{lo}}$ phenotype. Nevertheless, the slightly different SSC properties of atrial and ventricular cells may suggest differences in the cellular composition of the $\mathrm{ALDH}^{\mathrm{br}}$ population depending on the cardiac chamber of origin. This issue remains to be addressed in future studies.

Freshly isolated ALDH ${ }^{\text {br }}$ cells exhibited a heterogeneous phenotype, reflecting a mixture of different cell types (Figures 3 and 4 ). This population was significantly enriched for cells expressing Sca-1, the early hematopoietic and EPC marker CD34, the MSC marker CD90, the hyaluronic acid receptor CD44, and vascular cell adhesion molecule-1 (CD106). CD44 and CD106 were the most highly enriched markers in the $\mathrm{ALDH}^{\text {br }}$ versus $\mathrm{ALDH}^{\mathrm{dim}}$ population (34.3 and 24.1 fold-increases, resp.). Previous studies associated CD44 and
CD106 expression with MSCs [39]. However, a recent study showed that freshly isolated murine and human BM stromal cells did not express CD44 and CD106, but they could acquire expression of these antigens in vitro [40]. $\mathrm{CD} 44^{+} \mathrm{CD} 24^{-}$ cells have been proposed to represent cancer stem cells in breast cancer [41], as well as in head and neck squamous cell carcinoma (the latter includes an $\mathrm{ALDH} 1^{+} \mathrm{CD} 44^{+} \mathrm{CD} 24^{-}$ stem cell subset) [42]. In the present study, ALDH ${ }^{\text {very-br }}$ cells were highly enriched for Sca-1 and CD90, but only moderately so for $\mathrm{CD} 34$, possibly reflecting a predominant MSC-like subpopulation with fewer hematopoietic/vascular progenitors compared to $\mathrm{ALDH}^{\text {br }}$ cells with lower levels of ALDH activity. The ALDH ${ }^{\text {br }}$ population was significantly depleted for common leukocyte antigen (CD45), the endothelial marker CD31, and CD38 (an antigen expressed by the differentiating progeny of $\mathrm{CD} 34^{+} \mathrm{CD} 38^{-}$hematopoietic/vascular progenitors). ALDH ${ }^{\text {very-br }}$ cells were further depleted for lineage differentiation markers compared to $\mathrm{ALDH}^{\mathrm{br}}$ cells. Thus, the $\mathrm{ALDH}^{\mathrm{br}}$ population was enriched for MSC-like progenitors and depleted for differentiating hematopoietic and endothelial cells.

Purified $\mathrm{ALDH}^{\mathrm{br}}$ atrial cells could be expanded ex vivo, whereas their $\mathrm{ALDH}^{\mathrm{dim}}$ counterparts could not. Because the atrial population contained more $\mathrm{ALDH}^{\text {br }}$ cells than the ventricular population, atrial cells were used for the purification of $\mathrm{ALDH}^{\mathrm{br}}$ cells in order to shorten the sorting procedure, and therefore to attenuate cell damage. Purified 

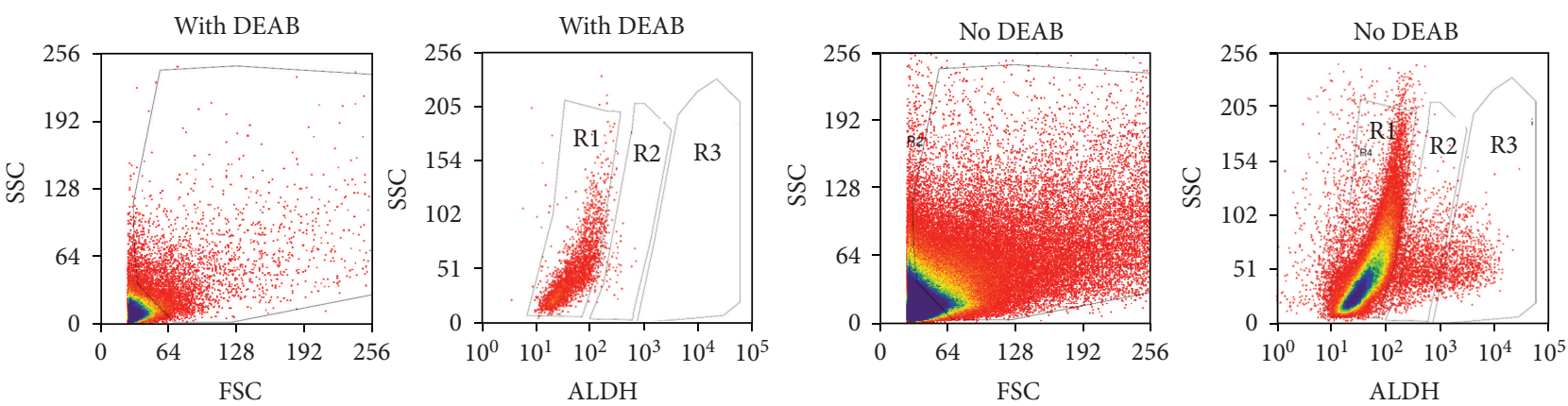

(a)

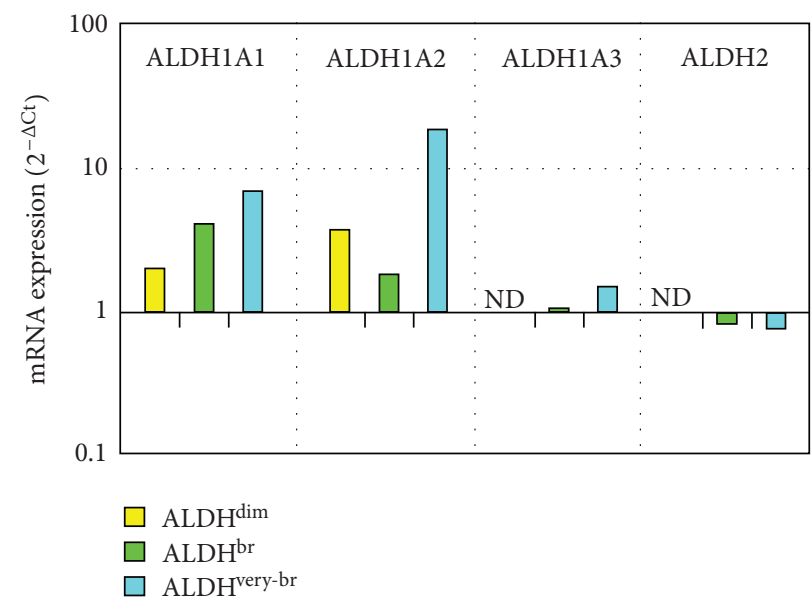

(b)

FIGURE 5: Real time RT-PCR analysis of gene transcripts of selected ALDH isoforms (a). FAC sorting of freshly isolated ALDH ${ }^{\text {dim }}$ (R1), $\mathrm{ALDH}^{\text {br }}$ (R2), and $\mathrm{ALDH}^{\text {very-br }}$ (R3) atrial cells from 8-week-old mice (b). RT-PCR analysis of ALDH1A1, ALDH1A2, ALDH1A3, and $\mathrm{ALDH} 2$ gene transcripts (data are $2^{-\triangle \mathrm{Ct}}$ values using GUSB as a reference gene). ND: not detectable.

$\mathrm{ALDH}^{\mathrm{br}}$ cells formed a few plastic-adherent colonies in each culture well. Each colony apparently originated from a single cell, providing observational evidence of clonal expansion, even though this was not firmly established using a clonogenic assay. The mechanism responsible for the selective growth of $\mathrm{ALDH}^{\mathrm{br}}$ cells in culture remains unclear. To initially address this question, we determined the respective contributions of selected ALDH isozymes to ALDH activity, measured by Aldefluor stain. Fifteen ALDH isozymes have been described in mice [43] and 19 in human $[9,44]$. They play different biological roles that vary among cell types and species. ALDH1A1, the ALDH isozyme most highly overexpressed in human BM ALDH ${ }^{\text {br }}$ versus ALDH ${ }^{\mathrm{dim}}$ cells [44], was believed to be responsible for ALDH activity, measured by Aldefluor stain, in BM and other tissues. However, recent findings in genetically modified mice demonstrated that ALDH1A1 was dispensable for stem cell function in the mouse hematopoietic and nervous systems [45]. ALDH1 was identified as a marker of normal and malignant human mammary stem cells and a predictor of poor clinical outcome in breast cancer [28]. In a distinct study, shRNA knock-down data indicated that ALDH1A3, not ALDH1A1, correlated best with ALDH activity in breast cancer stem cells [46]. ALDH1A1 and ALDH3A1 were highly expressed in nonsmall cell lung cancer, and knock-down of these ALDH isoforms were associated with in vitro functional changes in the proliferation and motility of these cells [47]. Human ALDH1A1, ALDH1A2, ALDH1A3, and ALDH8A1 function in retinoic acid cell signaling via retinoic acid production by oxidation of all-trans-retinal and 9-cis-retinal $[6,46]$. ALDH2, a mitochondrial isozyme that mediates both the detoxification of reactive aldehydes and the bioactivation of nitroglycerin to nitric oxide, mediates cytoprotection in the heart [48]. Recent data indicated that the aldehydeoxidizing activity of mouse HSCs, measured by Aldefluor stain, was due to the ALDH2 isozyme and correlated with protection against acetaldehyde toxicity [49]. Mice deficient in both Fanconi anemia pathway-mediated DNA repair and acetaldehyde detoxification showed more than a 600 -fold reduction in the HSC pool.

Based on these reports, we measured the expression of ALDH1A1, ALDH1A2, ALDH1A3, and ALDH2 gene transcripts in purified $\mathrm{ALDH}^{\mathrm{dim}}, \mathrm{ALDH}^{\mathrm{br}}$, and $\mathrm{ALDH}^{\text {very-br }}$ cardiac-derived cells (Figure 5). Expression of ALDH1A1 was lowest in $\mathrm{ALDH}^{\mathrm{dim}}$ cells, intermediate in $\mathrm{ALDH}^{\text {br }}$ cells, and highest in $\mathrm{ALDH}{ }^{\text {very-br }}$ cells $\left(2^{-\Delta \Delta \mathrm{Ct}}=3.5 \mathrm{using}\right.$ 

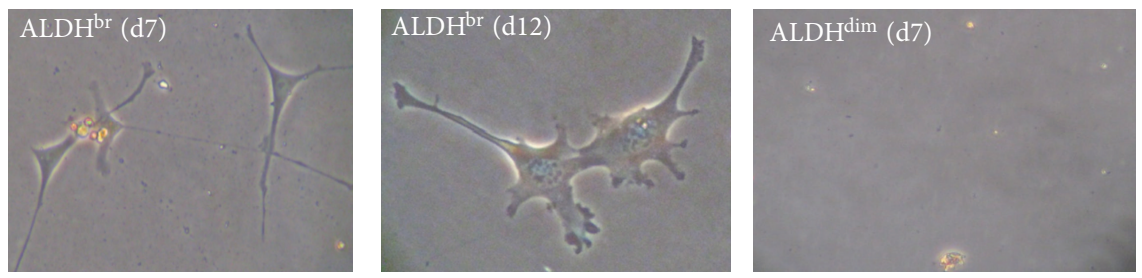

(a)

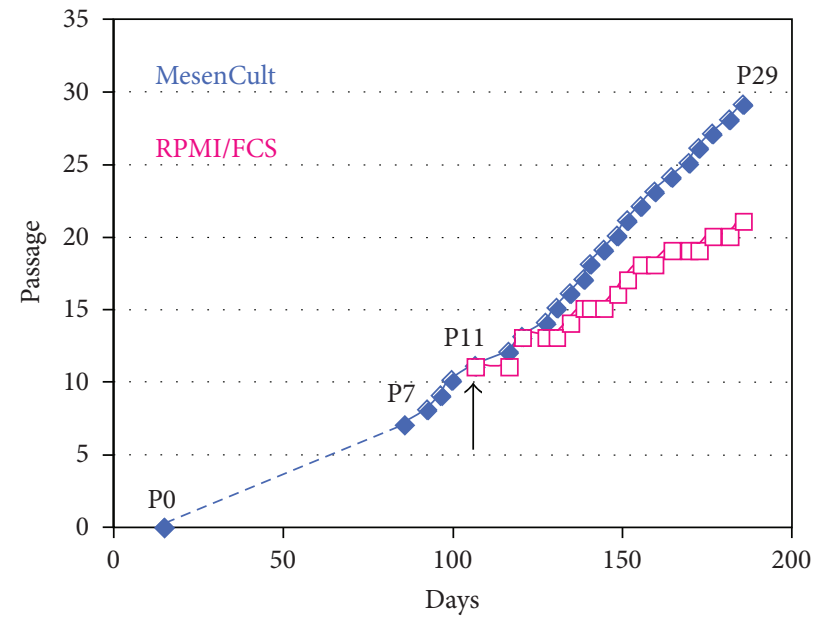

(b)

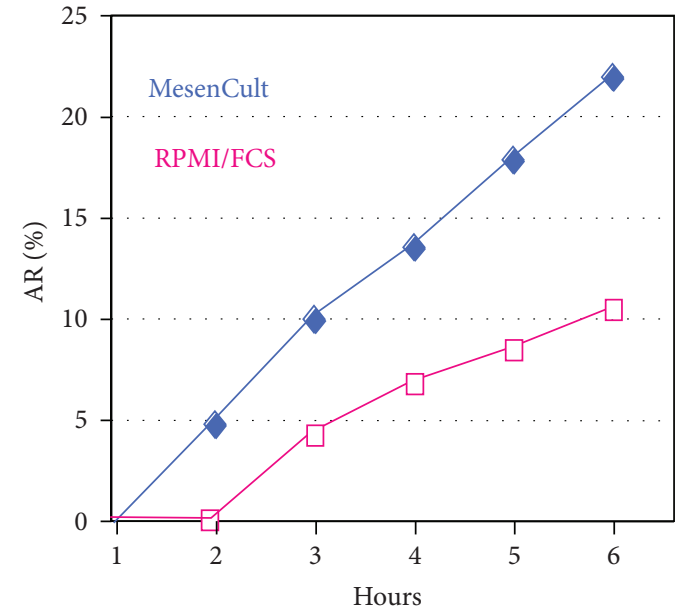

(c)

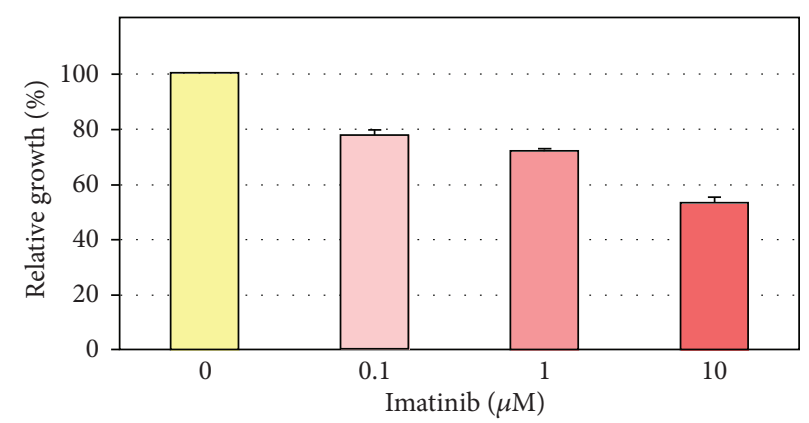

(d)

Figure 6: Ex vivo culture-expansion of $\mathrm{ALDH}^{\mathrm{br}}$ atrial cells (a). Photomicrographs of ALDH ${ }^{\text {br }}$ cells cultured for 7 and 12 days in MesenCult medium. ALDH ${ }^{\text {dim }}$ cells were dead by day 7 (b). Growth curves of cells in different growth media. Cells were cultured in MesenCult medium up to P10 (arrow). From P11 on, MesenCult was either maintained or replaced by RPMI/FCS. Symbols (squares and diamonds) indicate cell passage (no data available from P1 to P7; dotted line) (c). AlamarBlue assay on cells (P40) cultured in either MesenCult or RPMI/FCS (AR\%: percent AlamarBlue reduction) (d). Dose-response study of Imatinib with respect to growth inhibition of expanded ALDH ${ }^{\text {br }}$ cells (P25).

$\mathrm{ALDH}^{\mathrm{dim}}$ as a reference population). By contrast, expression of ALDH1A2 was lowest in $\mathrm{ALDH}^{\text {br }}$ cells, intermediate in $\mathrm{ALDH}^{\mathrm{dim}}$ cells, and highest in $\mathrm{ALDH}^{\text {very-br }}$ cells $\left(2^{-\Delta \Delta \mathrm{Ct}}=\right.$ 4.9 versus $\left.A L D H^{\text {dim }}\right)$. Expression of ALDH1A3 and ALDH2 was detectable in $\mathrm{ALDH}^{\text {br }}$ and $\mathrm{ALDH}^{\text {very-br }}$ cells, albeit at lower levels compared to ALDH1A1 and ALDH1A2, but not in $\mathrm{ALDH}^{\mathrm{dim}}$ cells. Thus, ALDH1A1 correlated best with ALDH activity; however, contributory roles of ALDH1A2, ALDH1A3, and ALDH2 to ALDH activity are possible based on our data. While the present analysis included the four ALDH isoforms most often associated with ALDH activity in previous reports [44-49], it should be completed to include the remaining ALDH isoforms. In addition, shRNA knockdown experiments are needed for identifying the ALDH isozyme(s) responsible for ALDH activity in these cells.

Recent data suggested BM and UCB ALDH ${ }^{\text {br }}$ cells might repair ischemic tissues in vivo by releasing angiogenic factors [30-32]. A comparison of gene expression profiles by $\mathrm{ALDH}^{\text {br }}$ and $\mathrm{ALDH}^{\text {dim }}$ human BM cells identified three angiogenic factors (endoglin/CD105, ephrin B4, and angiopoietin-1) as the most highly overexpressed factors in $\mathrm{ALDH}^{\text {br }}$ versus ALDH ${ }^{\text {dim }}$ cells (fold-changes: 66.9, 64.6, and 
ALDH ${ }^{\text {br }}$ cells in MesenCult medium
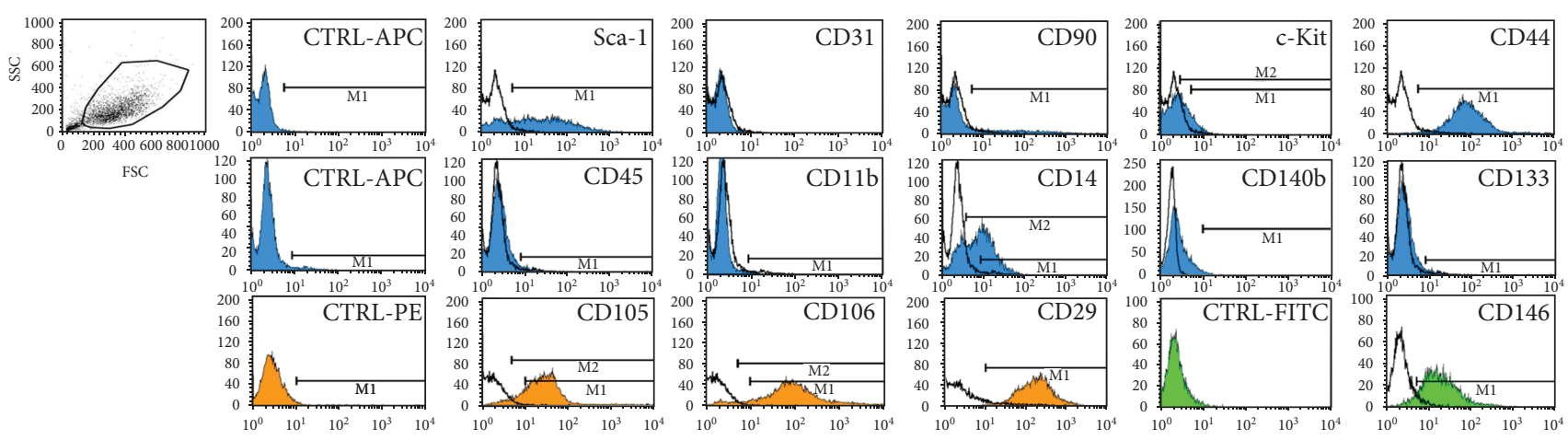

(a)

ALDH $^{\text {br }}$ cells in RPMI/FCS medium
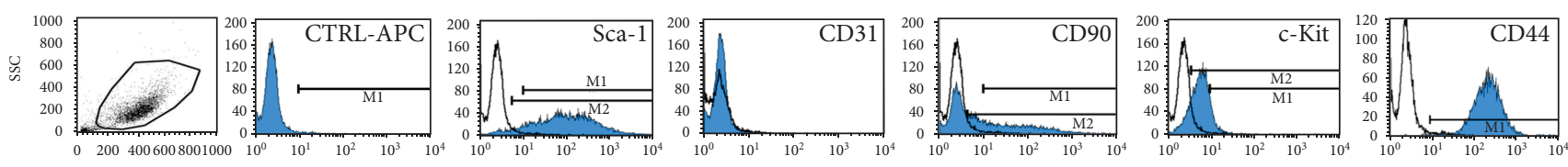

FSC
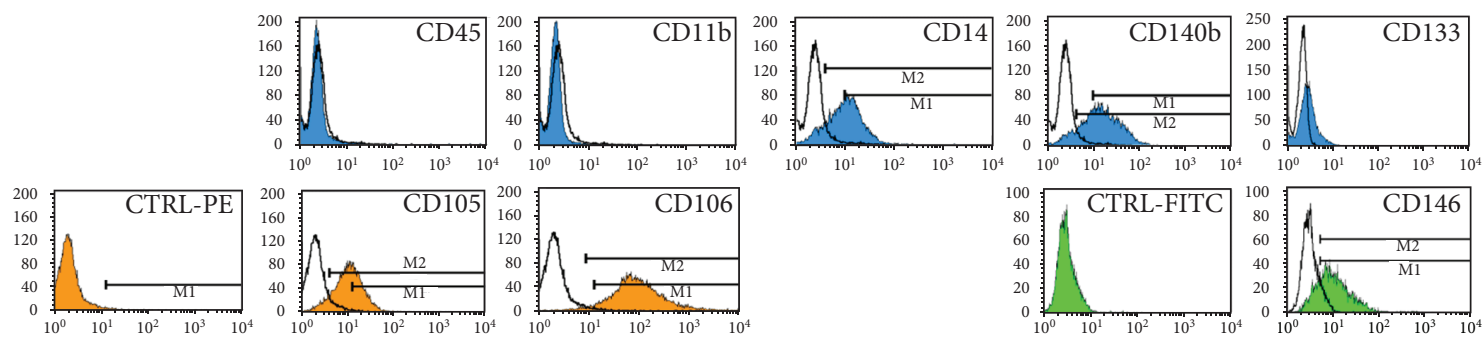

(b)

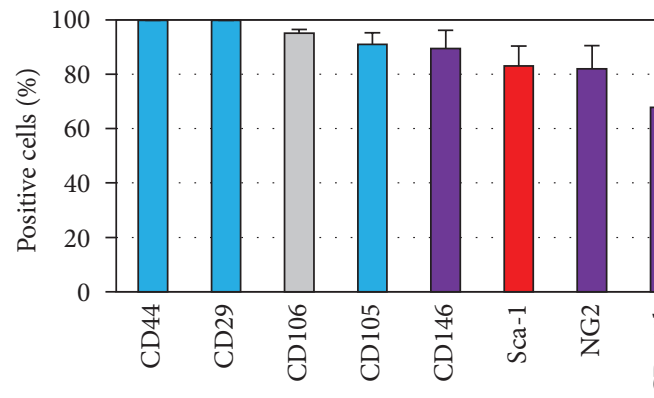

$\square$ MSC markers

$\square$ Hematopoetic markers

- Pericyte markers

(c)
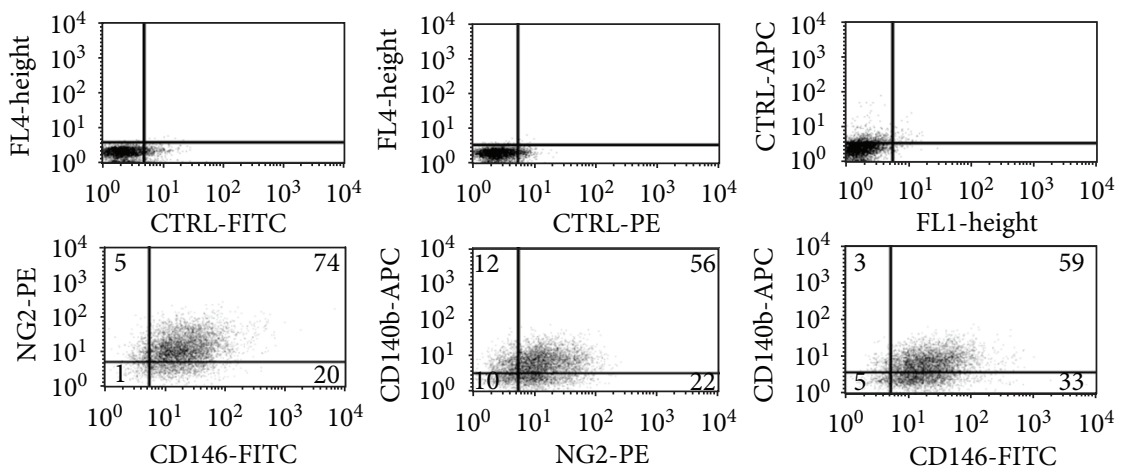

(d)

FIgURE 7: Continued. 

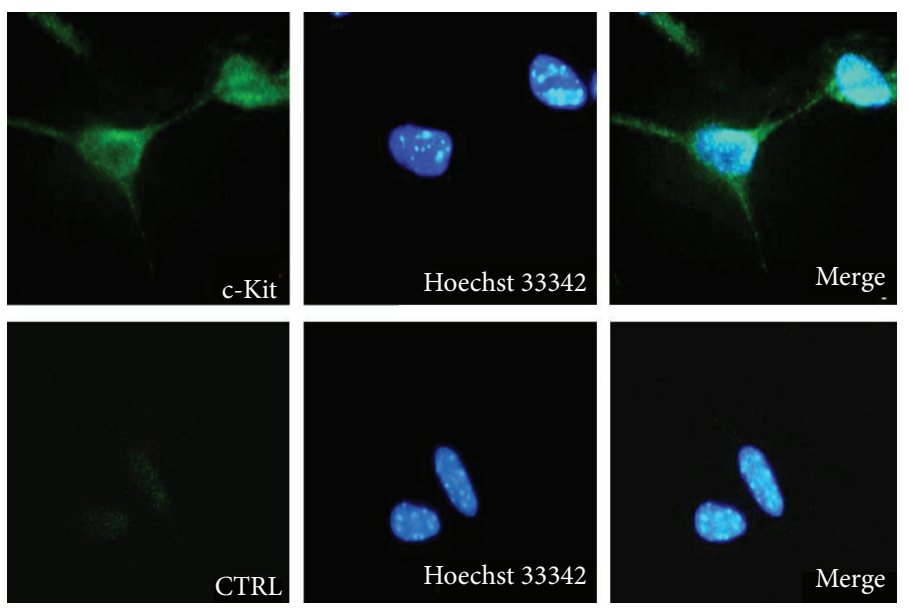

(e)

Figure 7: (a)/(b) Flow cytometric analysis of $\mathrm{ALDH}^{\text {br }}$ atrial cells expanded in MesenCult (P11) or RPMI/FCS medium (P13). (c) Percentages of cells (mean, SD) expressing the indicated markers (data from 3 separate experiments in MesenCult). (d) Two-color analysis of pericyte/perivascular markers (NG2, CD146, and CD140b; numbers are cell percentages). (e) c-kit immunostaining of expanded ALDH ${ }^{\text {br }}$ cells. Nuclear staining with Hoechst 33342 (CTRL, secondary Ab only as a control).

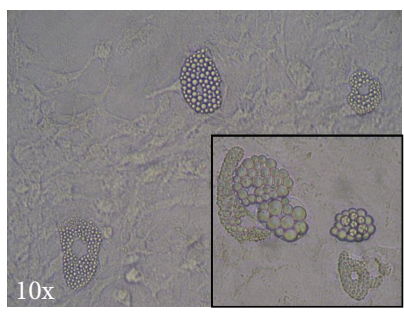

(a)

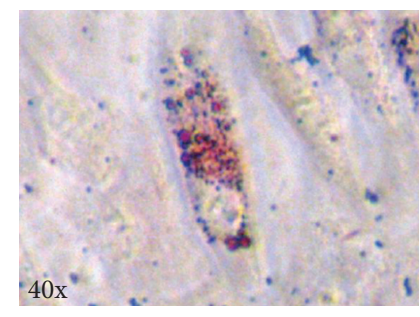

(b)

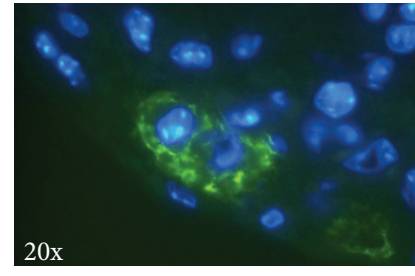

(c)

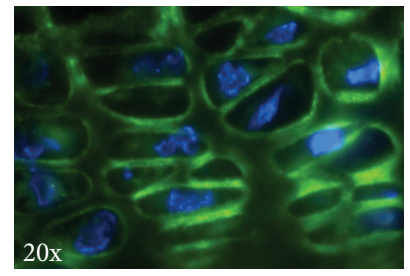

(d)

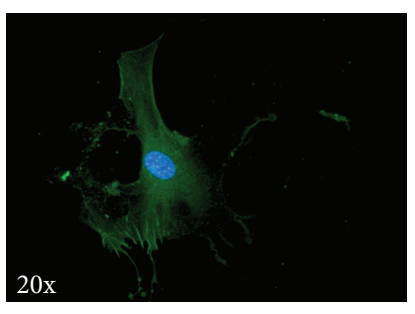

(e)

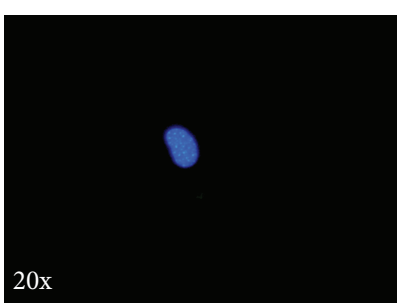

(f)

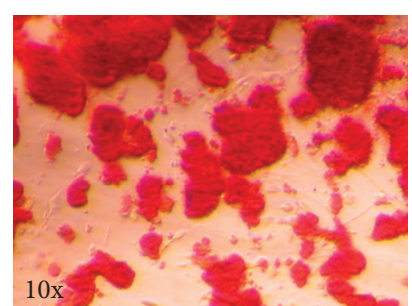

$(\mathrm{g})$

Figure 8: Differentiation potential of expanded $\mathrm{ALDH}^{\text {br }}$ cells. (a) Photomicrograph showing intracellular fat droplets in cells cultured in adipogenic medium. (b) Positive Oil Red-O staining of intracellular droplets. (c) Positive type-II collagen immunostaining (green) in cells cultured in chondrogenic medium. (d) Type-II collagen immunostaining of leg cartilage, as a positive control. (e) Positive sarcomeric $\alpha$ actinin immunostaining (green) of an $\mathrm{ALDH}^{\text {br }}$ cell (the protein does not show clear sarcomeric distribution). (f) Corresponding negative control (secondary Ab only). (g) Positive Alizarin Red staining of expanded ALDH ${ }^{\text {br }}$ cells cultured in osteogenic medium.

7.0, resp.) [44]. We therefore measured mRNA expression of these angiogenic genes in cardiac-derived cells but found no correlation with ALDH activity (besides the preliminary observation that angiopoietin-1 transcripts were detectable in $\mathrm{ALDH}^{\text {br }}$ and ALDH ${ }^{\text {very-br }}$ cells but not in $\mathrm{ALDH}^{\text {dim }}$ cells). The angiogenic potential of cardiac-derived $\mathrm{ALDH}^{\text {br }}$ cells remains to be addressed in future studies. Previous reports showed that $\mathrm{ALDH}^{\mathrm{br}}$ MSCs from human UCB were more responsive to hypoxia than their ALDH ${ }^{\text {dim }}$ counterparts, with upregulation of Flt-1, CXCR4, and angiopoietin-2 [50]. The same group reported that $\mathrm{ALDH}^{\mathrm{dim}}$ EPCs, but not $\mathrm{ALDH}^{\mathrm{br}}$ EPCs, from human UCB upregulated hypoxia-inducible factor proteins as well as VEGF, CXCR4, and GLUT-1 mRNAs under hypoxic conditions [22]. The introduction of ALDH ${ }^{\mathrm{dim}}$ EPCs significantly reduced ischemic tissue damage in a mouse flap model, whereas ALDH ${ }^{\text {br }}$ EPCs were ineffective. 
These findings suggested varying angiogenic activities of $\mathrm{ALDH}^{\text {br }}$ cells depending on the cell type studied.

In the present study, plastic-adherent cell clusters grew rapidly and could be passaged more than 40 times (later passages were not tested). Growth rates in complete MesenCult medium were higher than in RPMI/FCS (Figure 6). Imatinib, which selectively inhibits PDGFRB (CD140b) and c-kit tyrosine kinases with a similar potency [51], reduced cell proliferation in a dose-dependent manner. Expression of PDGFRB and c-kit was detected in subsets of expanded cells. The effect of Imatinib might reflect a role for PDGFRB and/or c-kit in the proliferation of these cells. Previous studies showed that ALDH ${ }^{\text {br }}$ MSCs from UCB proliferated more than their ALDH ${ }^{\mathrm{dim}}$ counterparts [50], whereas ALDH $^{\text {dim }}$ EPCs proliferated more than ALDH ${ }^{\text {br }}$ EPCs [22]. Proliferative properties correlated with angiogenic activities in these populations. Again, the association of ALDH and proliferation may vary among cell types. The immunophenotype of expanded ALDH ${ }^{\text {br }}$ cardiac cells resembled that of freshly isolated ALDH ${ }^{\text {br }}$ cells (Figure 7). Positive stains for pericytic markers (NG2, CD146, and CD140b) were consistent with a MSC-like phenotype. It has been shown that perivascular cells of arterioles and capillaries from many tissues express pericytic markers and show MSClike and angiogenic features [52-54]. In the present study, expanded cells stained positive for sarcomeric $\alpha$-actinin, a cardiac marker, and exhibited differentiation potential along multiple mesenchymal lineages. Previous data showed that aldehyde dehydrogenase activity did not increase the chondrogenic potential of human adipose-derived adult stem cells [55]. Recent reports showed that human BM contained ALDH ${ }^{\text {br }}$ multipotential mesenchymal progenitor cells (MPCs), besides ALDH ${ }^{\text {br }}$ HSCs and EPCs $[12,56]$. These MPCs strongly expressed ALDH, expressed embryonic markers not present in mesenchymal cells, and could be differentiated to microvascular endothelial cells and mesenchymal cells. The latter lacked strong ALDH expression and could not be induced to back differentiate into MPCs.

We observed a loss of $\mathrm{ALDH}^{\text {br }}$ cells with cell passage (e.g., only $11 \%$ of cells at passage 7 were still $\mathrm{ALDH}^{\mathrm{br}}$ ). Whether this loss of ALDH ${ }^{\text {br }}$ cells with time reflected cellular adaptation to ex vivo conditions or the replacement of early progenitors by a progeny of late progenitor and precursor cells remains unclear. The observation that growth rates and the immunophenotype remained stable for more than 30 and 10 passages, respectively, seemed not to support cell differentiation. ALDH as a stem/progenitor cell marker has chiefly been used in freshly isolated cells and tissues, as opposed to expanded cell populations. The significance of ALDH in culture-expanded cells is unclear, as is its contribution to stem cell fate. The aforementioned observation that the mesenchymal cell progeny of ALDH ${ }^{\text {br }}$ human BM cells lacked strong ALDH expression and could not be induced to back differentiate into MPCs suggested a parallel loss of ALDH expression and cell potency [56].

In conclusion, cardiac-derived $\mathrm{ALDH}^{\text {br }}$ cells are progenitor cells with MSC-like phenotype and function and with superior ex vivo growth characteristics compared to their ALDH ${ }^{\mathrm{dim}}$ counterparts. Further studies are needed for assessing the regenerative potential of these cells in animal models of heart disease.

\section{Acknowledgments}

This work is supported by the Swiss Heart Foundation (Berne, Switzerland), the Fondation Vaudoise de Cardiologie (Lausanne, Switzerland), the Cecilia Augusta Foundation (Lugano, Switzerland), the METIS Foundation Sergio Mantegazza (Lugano, Switzerland), and the "Fondazione per la ricerca sulla trasfusione e sui trapianti" (Lugano, Switzerland).

\section{References}

[1] H. Oh, S. B. Bradfute, T. D. Gallardo et al., "Cardiac progenitor cells from adult myocardium: homing, differentiation, and fusion after infarction," Proceedings of the National Academy of Sciences of the United States of America, vol. 100, no. 21, pp. 12313-12318, 2003.

[2] A. P. Beltrami, L. Barlucchi, D. Torella et al., "Adult cardiac stem cells are multipotent and support myocardial regeneration," Cell, vol. 114, no. 6, pp. 763-776, 2003.

[3] K. L. Laugwitz, A. Moretti, J. Lam et al., "Postnatal isl1+ cardioblasts enter fully differentiated cardiomyocyte lineages," Nature, vol. 433, no. 7026, pp. 647-653, 2005.

[4] K. L. Laugwitz, A. Moretti, J. Lam et al., "Erratum: Postnatal isl1+ cardioblasts enter fully differentiated cardiomyocyte lineages," Nature, vol. 446, no. 7138, p. 934, 2007.

[5] J. Cai, M. L. Weiss, and M. S. Rao, "In search of "stemness"', Experimental Hematology, vol. 32, no. 7, pp. 585-598, 2004.

[6] A. E. Balber, "Concise review: aldehyde dehydrogenase bright stem and progenitor cell populations from normal tissues: characteristics, activities, and emerging uses in regenerative medicine," Stem Cells, vol. 29, no. 4, pp. 570-575, 2011.

[7] D. J. Laird, A. W. De Tomaso, and I. L. Weissman, "Stem cells are units of natural selection in a colonial ascidian," Cell, vol. 123, no. 7, pp. 1351-1360, 2005.

[8] D. J. Laird, A. W. De Tomaso, and I. L. Weissman, "Erratum: Stem cells are units of natural selection in a colonial ascidian," Cell, vol. 124, no. 3, pp. 647-648, 2006.

[9] S. A. Marchitti, C. Brocker, D. Stagos, and V. Vasiliou, "NonP450 aldehyde oxidizing enzymes: the aldehyde dehydrogenase superfamily," Expert Opinion on Drug Metabolism and Toxicology, vol. 4, no. 6, pp. 697-720, 2008.

[10] M. B. Kastan, E. Schlaffer, J. E. Russo, O. M. Colvin, C. I. Civin, and J. Hilton, "Direct demonstration of elevated aldehyde dehydrogenase in human hematopoietic progenitor cells," Blood, vol. 75, no. 10, pp. 1947-1950, 1990.

[11] R. W. Storms, A. P. Trujillo, J. B. Springer et al., "Isolation of primitive human hematopoietic progenitors on the basis of aldehyde dehydrogenase activity," Proceedings of the National Academy of Sciences of the United States of America, vol. 96, no. 16, pp. 9118-9123, 1999.

[12] T. Gentry, S. Foster, L. Winstead, E. Deibert, M. Fiordalisi, and A. Balber, "Simultaneous isolation of human BM hematopoietic, endothelial and mesenchymal progenitor cells by flow sorting based on aldehyde dehydrogenase activity: implications for cell therapy," Cytotherapy, vol. 9, no. 3, pp. 259-274, 2007. 
[13] J. P. Chute, G. G. Muramoto, J. Whitesides et al., "Inhibition of aldehyde dehydrogenase and retinoid signaling induces the expansion of human hematopoietic stem cells," Proceedings of the National Academy of Sciences of the United States of America, vol. 103, no. 31, pp. 11707-11712, 2006.

[14] D. A. Hess, T. E. Meyerrose, L. Wirthlin et al., "Functional characterization of highly purified human hematopoietic repopulating cells isolated according to aldehyde dehydrogenase activity," Blood, vol. 104, no. 6, pp. 1648-1655, 2004.

[15] R. W. Storms, P. D. Green, K. M. Safford et al., "Distinct hematopoietic progenitor compartments are delineated by the expression of aldehyde dehydrogenase and CD34," Blood, vol. 106, no. 1, pp. 95-102, 2005.

[16] D. A. Hess, L. Wirthlin, T. P. Craft et al., "Selection based on CD133 and high aldehyde dehydrogenase activity isolates longterm reconstituting human hematopoietic stem cells," Blood, vol. 107, no. 5, pp. 2162-2169, 2006.

[17] D. J. Pearce and D. Bonnet, "The combined use of Hoechst efflux ability and aldehyde dehydrogenase activity to identify murine and human hematopoietic stem cells," Experimental Hematology, vol. 35, no. 9, pp. 1437-1446, 2007.

[18] O. Christ, K. Lucke, S. Imren et al., "Improved purification of hematopoietic stem cells based on their elevated aldehyde dehydrogenase activity," Haematologica, vol. 92, no. 9, pp. 1165-1172, 2007.

[19] T. Gentry, E. Deibert, S. J. Foster, R. Haley, J. Kurtzberg, and A. E. Balber, "Isolation of early hematopoietic cells, including megakaryocyte progenitors, in the ALDH-bright cell population of cryopreserved, banked UC blood," Cytotherapy, vol. 9, no. 6, pp. 569-576, 2007.

[20] P. Fallon, T. Gentry, A. E. Balber et al., "Mobilized peripheral blood SSCloALDHbr cells have the phenotypic and functional properties of primitive haematopoietic cells and their number correlates with engraftment following autologous transplantation," British Journal of Haematology, vol. 122, no. 1, pp. 99-108, 2003.

[21] T. J. Povsic, K. L. Zavodni, F. L. Kelly et al., "Circulating progenitor cells can be reliably identified on the basis of aldehyde dehydrogenase activity," Journal of the American College of Cardiology, vol. 50, no. 23, pp. 2243-2248, 2007.

[22] M. Nagano, T. Yamashita, H. Hamada et al., "Identification of functional endothelial progenitor cells suitable for the treatment of ischemic tissue using human umbilical cord blood," Blood, vol. 110, no. 1, pp. 151-160, 2007.

[23] S. Corti, F. Locatelli, D. Papadimitriou et al., "Transplanted ALDHhiSSClo neural stem cells generate motor neurons and delay disease progression of nmd mice, an animal model of SMARD1," Human Molecular Genetics, vol. 15, no. 2, pp. 167-187, 2006.

[24] S. Corti, M. Nizzardo, M. Nardini et al., "Neural stem cell transplantation can ameliorate the phenotype of a mouse model of spinal muscular atrophy," Journal of Clinical Investigation, vol. 118, no. 10, pp. 3316-3330, 2008.

[25] K. Vauchez, J. P. Marolleau, M. Schmid et al., "Aldehyde dehydrogenase activity identifies a population of human skeletal muscle cells with high myogenic capacities," Molecular Therapy, vol. 17, no. 11, pp. 1948-1958, 2009.

[26] E. Jean, D. Laoudj-Chenivesse, C. Notarnicola et al., "Aldehyde dehydrogenase activity promotes survival of human muscle precursor cells," Journal of Cellular and Molecular Medicine, vol. 15, no. 1, pp. 119-133, 2011.
[27] E. H. Huang, M. J. Hynes, T. Zhang et al., "Aldehyde dehydrogenase 1 is a marker for normal and malignant human colonic stem cells (SC) and tracks SC overpopulation during colon tumorigenesis," Cancer Research, vol. 69, no. 8, pp. 3382-3389, 2009.

[28] C. Ginestier, M. H. Hur, E. Charafe-Jauffret et al., "ALDH1 is a marker of normal and malignant human mammary stem cells and a predictor of poor clinical outcome," Cell Stem Cell, vol. 1, no. 5, pp. 555-567, 2007.

[29] P. Marcato, C. A. Dean, D. Pan et al., "Aldehyde dehydrogenase activity of breast cancer stem cells is primarily due to isoform ALDH1A3 and its expression is predictive of metastasis," Stem Cells, vol. 29, no. 1, pp. 32-45, 2011.

[30] C. S. Sondergaard, D. A. Hess, D. J. Maxwell et al., "Human cord blood progenitors with high aldehyde dehydrogenase activity improve vascular density in a model of acute myocardial infarction," Journal of Translational Medicine, vol. 8, article 24, 2010.

[31] B. J. Capoccia, D. L. Robson, K. D. Levac et al., "Revascularization of ischemic limbs after transplantation of human bone marrow cells with high aldehyde dehydrogenase activity," Blood, vol. 113, no. 21, pp. 5340-5351, 2009.

[32] G. I. Bell, H. C. Broughton, K. D. Levac, D. A. Allan, A. Xenocostas, and D. A. Hess, "Transplanted human bone marrow progenitor subtypes stimulate endogenous islet regeneration and revascularization," Stem Cells and Development, vol. 21, no. 1, pp. 97-109, 2012.

[33] E. C. Perin, G. Silva, A. Gahremanpour et al., "A randomized, controlled study of autologous therapy with bone marrowderived aldehyde dehydrogenase bright cells in patients with critical limb ischemia," Catheterization and Cardiovascular Interventions, vol. 78, no. 7, pp. 1060-1067, 2011.

[34] E. C. Perin, G. V. Silva, Y. Zheng et al., "First randomized, controlled double-blind trial of transendocardial injection of autologous aldehyde dehydrogenase-bright stem cells," American Heart Journal, vol. 161, no. 6, pp. 1078-11087, 2011.

[35] E. C. Perin, G. V. Silva, Y. Zheng et al., "Randomized, doubleblind pilot study of transendocardial injection of autologous aldehyde dehydrogenase-bright stem cells in patients with ischemic heart failure," American Heart Journal, vol. 163, no. 3, pp. 415-421, 2012.

[36] K. Urbanek, D. Cesselli, M. Rota et al., "Stem cell niches in the adult mouse heart," Proceedings of the National Academy of Sciences of the United States of America, vol. 103, no. 24, pp. 9226-9231, 2006.

[37] A. Meinhardt, A. Spicher, M. E. Roehrich, I. Glauche, P. Vogt, and G. Vassalli, "Immunohistochemical and flow cytometric analysis of long-term label-retaining cells in the adult heart," Stem Cells and Development, vol. 20, no. 2, pp. 211-222, 2011.

[38] C. Castaldo, F. Di Meglio, D. Nurzynska et al., "CD117-positive cells in adult human heart are localized in the subepicardium, and their activation is associated with laminin- 1 and $\alpha 6$ integrin expression," Stem Cells, vol. 26, no. 7, pp. 1723-1731, 2008.

[39] M. Baddoo, K. Hill, R. Wilkinson et al., "Characterization of mesenchymal stem cells isolated from murine bone marrow by negative selection," Journal of Cellular Biochemistry, vol. 89, no. 6, pp. 1235-1249, 2003.

[40] H. Qian, K. Le Blanc, and M. Sigvardsson, "Primary mesenchymal stem and progenitor cells from bone marrow lack expression of CD44 protein," The Journal of Biological Chemistry, vol. 287, no. 31, pp. 25795-25807, 2012. 
[41] M. Al-Hajj, M. S. Wicha, A. Benito-Hernandez, S. J. Morrison, and M. F. Clarke, "Prospective identification of tumorigenic breast cancer cells," Proceedings of the National Academy of Sciences of the United States of America, vol. 100, no. 7, pp. 3983-3988, 2003.

[42] C. Chen, Y. Wei, M. Hummel et al., "Evidence for epithelialmesenchymal transition in cancer stem cells of head and neck squamous cell carcinoma," PLoS ONE, vol. 6, no. 1, Article ID e16466, 2011.

[43] Y. Alnouti and C. D. Klaassen, "Tissue distribution, ontogeny, and regulation of aldehyde dehydrogenase (Aldh) enzymes mRNA by prototypical microsomal enzyme inducers in mice," Toxicological Sciences, vol. 101, no. 1, pp. 51-64, 2008.

[44] H. Storrie White, L. Smith, T. Gentry, and A. E. Balber, "Mechanisms of action of human aldehyde dehydrogenase bright cells in therapy of cardiovascular diseases: expression analysis of angiogenic factors and aldehyde dehydrogenaseisozymes," Journal of Stem Cell Research. In press, http://dx.doi.org/10.4172/2157-7633.S1-001.

[45] B. P. Levi, Ö. H. Yilmaz, G. Duester, and S. J. Morrison, "Aldehyde dehydrogenase 1a1 is dispensable for stem cell function in the mouse hematopoietic and nervous systems," Blood, vol. 113, no. 8, pp. 1670-1680, 2009.

[46] P. Marcato, C. A. Dean, C. A. Giacomantonio, and P. W. K. Lee, "Aldehyde dehydrogenase its role as a cancer stem cell marker comes down to the specific isoform," Cell Cycle, vol. 10, no. 9, pp. 1378-1384, 2011.

[47] J. S. Moreb, H. V. Baker, L. J. Chang et al., "ALDH isozymes downregulation affects cell growth, cell motility and gene expression in lung cancer cells," Molecular Cancer, vol. 7, article 87, 2008.

[48] G. R. Budas, M. H. Disatnik, and D. Mochly-Rosen, "Aldehyde dehydrogenase 2 in cardiac protection: a new therapeutic target?" Trends in Cardiovascular Medicine, vol. 19, no. 5, pp. 158-164, 2009.

[49] J. I. Garaycoechea, G. P. Crossan, F. Langevin, M. Daly, M. J. Arends, and K. J. Patel, "Genotoxic consequences of endogenous aldehydes on mouse haematopoietic stem cell function," Nature, vol. 489, no. 7417, pp. 571-575, 2012.

[50] M. Nagano, K. Kimura, T. Yamashita et al., "Hypoxia responsive mesenchymal stem cells derived from human umbilical cord blood are effective for bone repair," Stem Cells and Development, vol. 19, no. 8, pp. 1195-1210, 2010.

[51] E. Buchdunger, C. L. Cioffi, N. Law et al., "Abl protein-tyrosine kinase inhibitor STI571 inhibits in vitro signal transduction mediated by c-Kit and platelet-derived growth factor receptors," Journal of Pharmacology and Experimental Therapeutics, vol. 295, no. 1, pp. 139-145, 2000.

[52] M. Crisan, S. Yap, L. Casteilla et al., "A perivascular origin for mesenchymal stem cells in multiple human organs," Cell Stem Cell, vol. 3, no. 3, pp. 301-313, 2008.

[53] P. Campagnolo, D. Cesselli, A. Al Haj Zen et al., "Human adult vena saphena contains perivascular progenitor cells endowed with clonogenic and proangiogenic potential," Circulation, vol. 121, no. 15, pp. 1735-1745, 2010.

[54] R. Katare, F. Riu, K. Mitchell et al., “Transplantation of human pericyte progenitor cells improves the repair of infarcted heart through activation of an angiogenic program involving microRNA-132," Circulation Research, vol. 109, no. 8, pp. 894-906, 2011.

[55] B. T. Estes, A. W. Wu, R. W. Storms, and F. Guilak, "Extended passaging, but not aldehyde dehydrogenase activity, increases the chondrogenic potential of human adipose-derived adult stem cells," Journal of Cellular Physiology, vol. 209, no. 3, pp. 987-995, 2006.

[56] M. Petrini, S. Pacini, L. Trombi et al., "Identification and purification of mesodermal progenitor cells from human adult bone marrow," Stem Cells and Development, vol. 18, no. 6, pp. 857-866, 2009. 

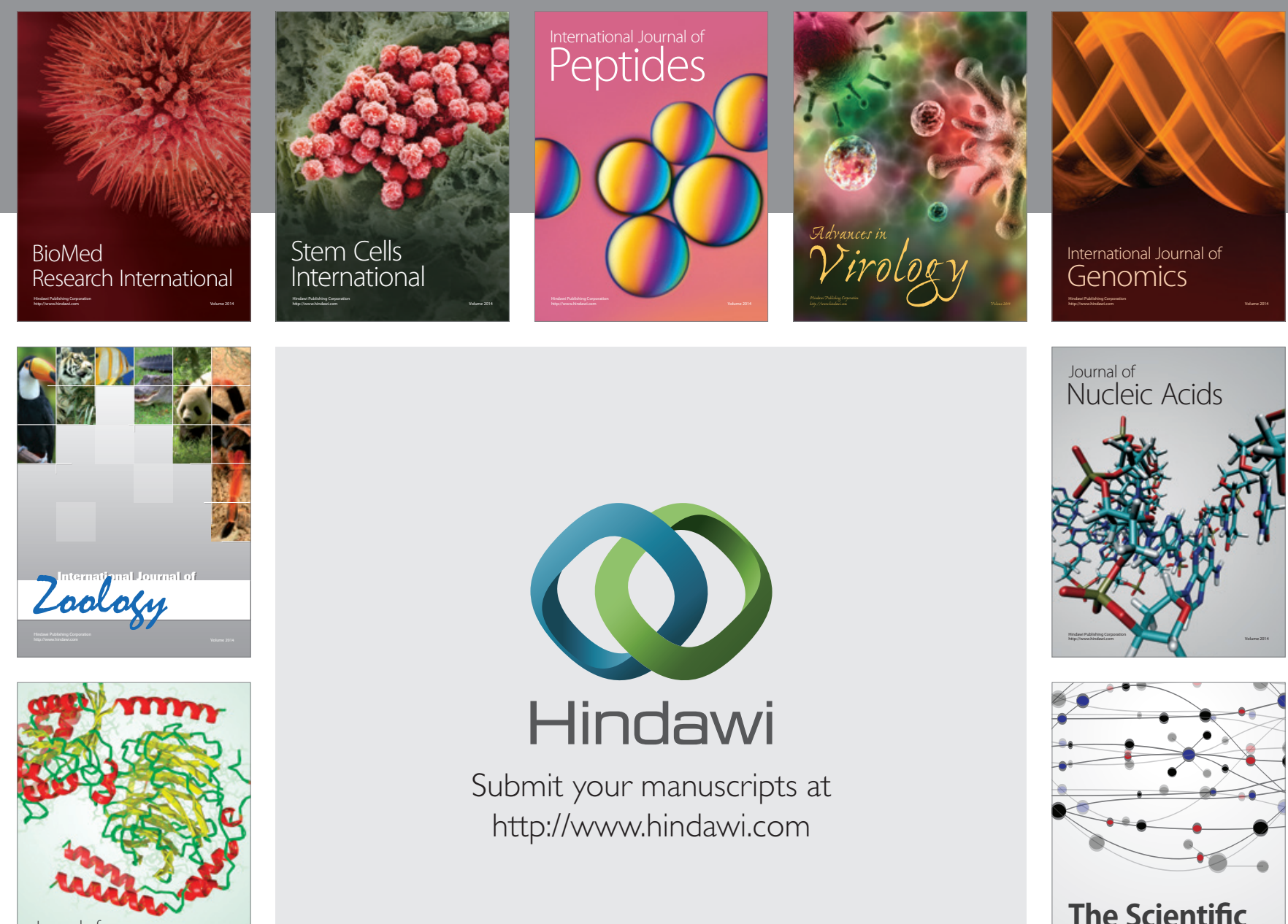

Submit your manuscripts at

http://www.hindawi.com

Journal of
Signal Transduction
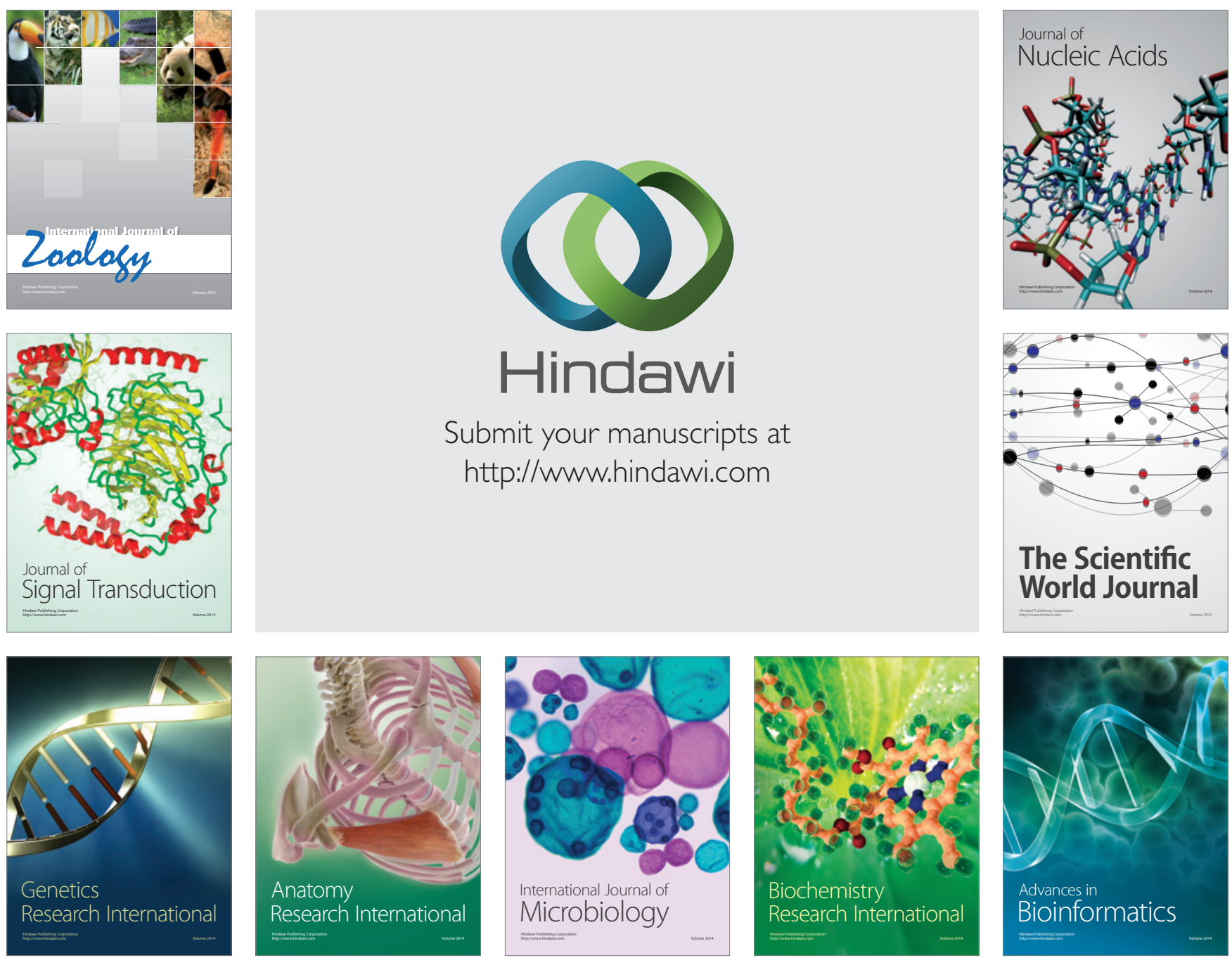

The Scientific World Journal
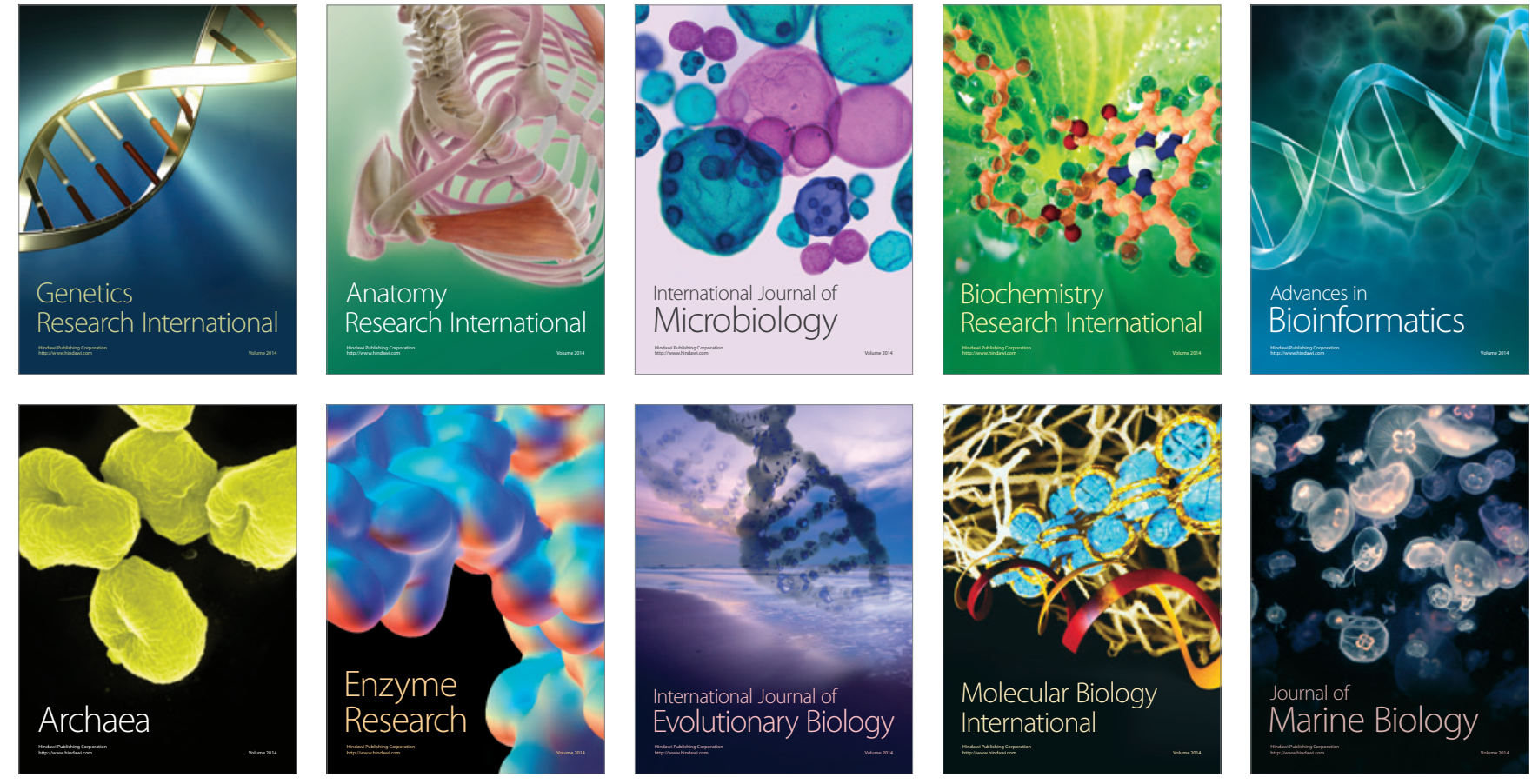